\title{
Branching processes of general Petri Nets
}

\author{
Jean-Michel Couvreur \\ Université d'Orléans, LIFO, Orléans, France, \\ jean-michel.couvreur@univ-orleans.fr
}

Denis Poitrenaud

Université Pierre et Marie Curie, LIP6, Paris, France,

denis.poitrenaud@lip6.fr

Pascal Weil ${ }^{*}$

CNRS, LaBRI, UMR 5800, F-33400 Talence, France

Univ. Bordeaux, LaBRI, UMR 5800, F-33400 Talence, France

pascal.weil@labri.fr

\begin{abstract}
We propose a new model of branching processes, suitable for describing the behavior of general Petri nets, without any finiteness or safeness assumption. In this framework, we define a new class of branching processes and unfoldings of a net $N$, which we call faithful. These coincide with the safe branching processes and unfoldings if $N$ is safe, or weakly safe as in [Engelfriet 1991], but not in general. However, faithful branching processes and processes satisfy the good order-theoretic properties which make the safe processes of safe nets so useful in practice, and which are known not to hold for the safe processes of a general net. Faithful processes represent therefore good candidates to generalize the theory of safe nets to the general case.
\end{abstract}

Keywords: General Petri nets; Unfoldings; Branching processes

\section{Introduction}

The study of the behavior of models of concurrency usually requires the definition of more abstract models. Within the framework of Petri nets, primarily two models were retained: labeled occurrence nets

Address for correspondence: Jean-Michel Couvreur, LIFO, Université d'Orléans, BP 6759, 45067 Orléans cedex 2, France.

* Supported by ANR project 2010 BLAN 020201 FREC. 
and event structures. Both models were proposed by Nielsen, Plotkin and Winskel in their foundational paper [13], in order to give a semantic of concurrency for safe Petri nets. The description of a safe Petri net execution is presented by a labeled causal net, called a process. Roughly speaking, causal nets are acyclic Petri nets whose places are without branching. In particular, their places and transitions are partially ordered, and this order, restricted to transitions, induces a partial order on the transition occurrences in the original Petri net. For the representation of conflicts, branching on places is allowed. This leads to the definition of labeled occurrence nets, called branching processes. The set of all the behaviors of the system can be captured by a single branching process, called the unfolding of the system, whose transitions are called events. By restricting the relations of causality and conflict to events, one obtains an event structure called the prime event structure. Since the publication of [13], there have been many attempts to extend these results to general Petri nets.

In these attempts, the focus has often been on using the same classes as in the case of safe nets, namely causal and occurrence Petri nets. Engelfriet [3] restricted his work to 1-valued arc-weights, initially 1-marked Petri nets, and he obtained good algebraic properties on branching processes (a structure of lattice) which led to the concept of unfolding.

For others (e.g. Best and Devillers [1], Meseguer, Montanari and Sassone [11]), markings take arbitrary values and tokens are individualized. As argued in [7], this is contrary to a pure multiset view of general nets, and the systems modelled by Petri nets rarely justify individualizing tokens. Similarly, Haar [5], pursuing Vogler's work [14], proposes an approach which aims at translating general nets into safe nets, by introducing a place for each reachable marking of original places. This not only considerably increases the size of the structure, but it also artificially introduces conflicts between transitions that access a given place. Thus it strongly departs from the intended semantic of nets.

In contrast with these approaches, Hoogers, Kleijn and Thiagarajan [7], propose a new event structure. In this so-called local event structure, tokens are not individualized. Their theory is complete for co-safe nets (see [7]), and it can be extended to the case of general nets. However, it does not present the expected properties in the general case.

In this paper, we propose a more net-theoretic approach, which does not impose to individualize the tokens, and which incorporates the solutions of $[1,3,11]$. Also, we do not make any finiteness assumption on our nets. Our formal framework allows us to identify a new structure, the faithful unfolding of a net, which allows for the good algebraic properties identified by Engelfriet [3], and which is applicable to general nets.

The starting point of our approach is an extension of the definitions of occurrence nets, which can be arbitrarily valued and non-safe, and of configurations, which are multisets of transitions (or events). Branching processes are defined as occurrence nets labeled by the elements of the original net. The differences between our definitions of occurrence nets and branching processes, and the classical definitions are discussed in Sections 3 and 4; it is important to note that these definitions coincide in the case of safe nets.

The set of branching processes of a net is equipped with a natural order relation, which leads to the definition of unfoldings as maximal branching processes. We identify two classes of branching processes and two types of unfoldings: weakly safe and faithful, and we show that every net admits a weakly safe and a faithful unfolding, which are unique up to isomorphism. The weakly safe unfolding coincides with those of $[1,3,11]$. The faithful unfolding is particularly interesting because of its order-theoretic properties. For safe nets, and more generally under the constraints on the net structure identified by Engelfriet in [3], there exists a unique unfolding, and the concepts of weakly safe and faithful unfolding 
coincide. In general however, the two unfoldings differ. The "universality" of the construction which associates a faithful unfolding to a general Petri net was already studied by Keller in a categorical setting [8], under various finiteness conditions (on the support of the initial marking and on the pre- and postsets of every transition).

In addition, we identify a partial order between the unfoldings of a net $N$ (taken up to isomorphism), and we show that the faithful unfolding and the weakly safe unfolding of $N$ are, respectively, the minimum and the maximum unfoldings with respect to that partial order.

Finally, we formalize the concept of process in our multiset context. Contrary to other works, our definition is not based on causal nets, but on the concept of a configuration. Again, it turns out that the safe case does not offer good enough properties (e.g., we cannot define the greatest lower bound of two processes). In contrast, the expected properties hold for faithful processes. This comes from the fact that a faithful process is represented in a unique way in the faithful unfolding.

The drawback of these algebraically and order-theoretically satisfactory structures is that the concepts of conflict and causality are not any more explicitly given by the model structure. That is, we lose a direct link with prime event structures, as in [7].

The paper is organized as follows. In Section 2, we fix the notation for nets, homomorphisms of nets and other fundamental objects. Section 3 discusses occurrence nets and configurations. In Section 4, generalized branching processes and unfoldings are introduced. The construction and the properties of the faithful and the weakly safe unfolding of a net are discussed in Section 5. Finally Section 6 presents the generalized notion of processes, and establishes the order-theoretic properties of faithful processes. Counter-examples of these properties for safe or weakly safe processes are also presented.

A preliminary version of these results appeared in the Proceedings of ATPN 2011 [2].

\section{Preliminaries}

We first summarize the basic notation and concepts used in this paper, concerning multisets and Petri nets. $\mathbb{N}$ denotes the set of non-negative integers.

\subsection{Notation}

Let $X$ be a set. A multiset over $X$ is a mapping $v: X \rightarrow \mathbb{N}$. Multisets are often represented as formal linear combinations, e.g. $v=a+2 b$ for $v(a)=1, v(b)=2, v(c)=0$ for all $c \in X \backslash\{a, b\}$, and it is also convenient to view them as vectors in $\mathbb{N}^{X}$. The support of the multiset $v$ is the set $\bar{v}=\{x \in X \mid$ $v(x)>0\}$. Note that the support of a multiset may be infinite. A multiset over a set $X$ can be naturally considered as a multiset over any superset of its support.

The operations of addition and subtraction of multisets over $X$ are defined componentwise, as on vectors (note however that negative coefficients are not allowed). An infinite sum of multisets $v=$ $\sum_{i \in I} v_{i}$ is said to be well-defined if for each $x \in X$, the sum $\sum_{i \in I} v_{i}(x)$ is finite. If $\sigma=x_{1} x_{2} \cdots$ is a finite sequence of elements of $X$, the characteristic vector of $\sigma$ is the multiset $\sigma=\sum_{i=1}^{|\sigma|} x_{i}$. Multisets are partially ordered by letting $v \leq w$ if $v(x) \leq w(x)$ for each $x \in X$.

If $X$ and $Y$ are sets, a mapping $h: X \rightarrow Y$ can sometimes be extended to multisets, $h: \mathbb{N}^{X} \rightarrow \mathbb{N}^{Y}$, by letting $h(v)=\sum_{x \in X} v(x) h(x)$ if the sum is well-defined (that is, if each $y \in Y$ has finitely many 
pre-images in the support of $v$ ). The mapping $h$ can also be extended to sequences of elements of $X$ by letting $h\left(x_{1} x_{2} \cdots\right)=h\left(x_{1}\right) h\left(x_{2}\right) \cdots$.

Let $\rightarrow$ be a relation on the set $X$, for instance the edge relation of a graph in which $X$ is the set of vertices. We denote by $\stackrel{*}{\rightarrow}$ (resp. $\stackrel{+}{\rightarrow}$ ), the reflexive and transitive closure (resp. transitive) of $\rightarrow$. We also use the following notation: if $Y \subseteq X$,

$$
\begin{aligned}
& { }^{\bullet} Y=\{x \in X \mid \exists y \in Y, x \rightarrow y\} \quad{ }^{*} Y=\{x \in X \mid \exists y \in Y, x \stackrel{*}{\rightarrow} y\} \\
& Y^{\bullet}=\{x \in X \mid \exists y \in Y, y \rightarrow x\} \quad Y^{*}=\left\{x \in X \mid \exists y \in Y, y^{*} x\right\} .
\end{aligned}
$$

If $Y=\{y\}$, we write simply ${ }^{\bullet} y,{ }^{*} y, y^{\bullet}$ and $y^{*}$.

When the graph $(X, \rightarrow)$ is acyclic (i.e, $x \stackrel{+}{\rightarrow} x$ never holds, for any $x \in X$ ), the relation $\stackrel{*}{\rightarrow}$ forms a partial order on a set $X$.

If $(X, \leq)$ is a partially ordered set and if $Y \subseteq X$, we say that $Y$ is an order ideal if $x \in Y$ whenever $x \leq y$ for some $y \in Y$. Moreover, we say that $x$ is a lower (resp. upper) bound of $Y$ if $x \leq y$ (resp. $y \leq x$ ) for each element $y \in Y$. The greatest lower bound (resp. least upper bound) of $Y$, if it exists, is denoted by $\inf (Y)($ resp. $\sup (Y))$. If any two elements of $X$ admit a greatest lower bound and a least upper bound, $X$ is called a lattice. It is a complete lattice if any subset of $X$ admits a greatest lower and a least upper bound.

\subsection{Petri net}

A Petri net, or simply a net, is a tuple $N=\left(P, T\right.$, Pre, Post, $\left.m_{0}\right)$ consisting of two disjoint sets $P$ and $T$ whose elements are called places and transitions, two multisets Pre and Post over $P \times T$ (sometimes called the flow functions), and a multiset $m_{0}$ over $P$ called the initial marking. A marking of $N$ is any multiset over $P$. If $t$ is a transition, the pre-condition of $t$, written $\operatorname{Pre}(t)$, is the marking $\operatorname{Pre}(\cdot, t)$. Similarly, the post-condition of $t$, written Post $(t)$ is the marking Post $(\cdot, t)$.

Note that we don't make any finiteness assumption: $P$ or $T$ may be infinite, as well as the support of the initial marking or of the pre- and post-condition of a transition. A net may be viewed as a labelled bipartite graph (the graphical representation of $N$ ) as follows: we can identify $N$ with the labeled graph $\left(P, T, \rightarrow, m_{0}\right)$, where places and transitions are the two disjoint sets of nodes, there is an edge $p \stackrel{\operatorname{Pre}(p, t)}{\longrightarrow} t$

(resp. $t \stackrel{\operatorname{Post}(p, t)}{\longrightarrow} p$ ) between the place $p$ and the transition $t$ if $\operatorname{Pre}(p, t)(\operatorname{resp} . \operatorname{Post}(p, t))$ is non-zero, and $m_{0}$ is a labelling function of the places, traditionally depicted by the presence of $m_{0}(p)$ tokens in place $p$.

Example 2.1. Figure 1 represents a net modelling a reader/writer system allowing at most three concurrent readers. We use the classical graphical representation of Petri nets, where places are drawn as circles and transitions as thin rectangles, where the initial marking is indicated by the corresponding number of tokens in each place, and where pre- and post-conditions are indicated by labels on edges. The initial marking of place $S$ allows at most three readers to be simultaneously in place $C_{0}$. Only one writer at a time can access place $C_{1}$, and when she is there, no reader may be in place $C_{0}$.

If $t$ is a transition and $m$ is a marking of $N$, we say that $t$ is enabled by $m$, written $m[t\rangle$, if $\operatorname{Pre}(t) \leq m$. Firing the transition $t$ in $m$ produces the marking

$$
m^{\prime}=m+\operatorname{Post}(t)-\operatorname{Pre}(t)
$$




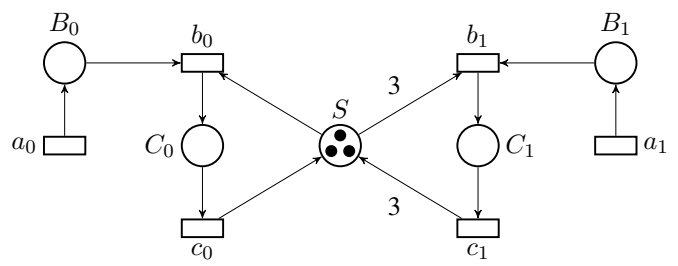

Figure 1. Graphical representation of a net modeling a reader/writer system

and we write $m[t\rangle m^{\prime}$. The firing equation is extended inductively to any sequence of transitions: if $\varepsilon$ is the empty sequence, we let $m[\varepsilon\rangle m$; if $\sigma$ is a sequence of transitions and if $t$ is a transition, then $m[\sigma t\rangle m^{\prime}$ if there exists a marking $m^{\prime \prime}$ such that $m[\sigma\rangle m^{\prime \prime}$ and $m^{\prime \prime}[t\rangle m^{\prime}$. It is easily verified that if $\sigma=t_{1} t_{2} \cdots t_{n}$ and $m[\sigma\rangle m^{\prime}$, then

$$
m^{\prime}=m+\sum_{i=1}^{n} \operatorname{Post}\left(t_{i}\right)-\sum_{i=1}^{n} \operatorname{Pre}\left(t_{i}\right) .
$$

(extended firing equation)

A transition, or sequence of transitions, is said to be firable if it is enabled by the initial marking. A marking $m$ is called reachable if there exists a finite sequence of transitions $\sigma$ such that $m_{0}[\sigma\rangle m$.

A transition $t$ is called spontaneous if $\operatorname{Pre}(t)=0$, that is, ${ }^{\bullet} t=\emptyset$. A place or a transition $x$ is called isolated if $\bullet=x^{\bullet}=\emptyset$.

In this paper we will use the following properties of nets. Recall that a marking $m$ is safe if $m(p) \leq 1$ for each place $p$. The net $N$ is said to be

- weakly safe if the initial marking $m_{0}$ and the pre- and post-condition $\operatorname{Pre}(t)$ and $\operatorname{Post}(t)$ of each transition $t$ are safe markings;

- safe if each reachable marking is safe;

- quasi-live if every transition is enabled by a reachable marking;

- acyclic if the graph representing $N$ is acyclic; in that case, the induced partial order on the set of places and transitions is written $\leq$.

These notions are classical, see [12], with the exception of weakly safe nets: our definition differs from Engelfriet's [3] in the fact that we allow spontaneous transitions. It is interesting to note that weak safeness is a property that can be easily verified upon reading the definition of a Petri net, whereas deciding safeness requires computing a transitive closure, a notoriously high complexity procedure.

Lemma 2.2. Every safe and quasi-live Petri net is weakly safe.

Proof. If $N$ is safe, then the initial marking $m_{0}$ is safe. We now verify that the pre- and post-conditions of a transition $t$ of $N$ are safe markings. As we are assuming that $t$ is enabled by some reachable marking, and therefore by a safe marking, the marking Pre $(t)$ must be safe. For the same reason, since $t$ appears in a sequence of transitions enabled by the initial marking, leading to a safe marking, the marking Post $(t)$ must be safe as well. This concludes the proof. 
For further reference, we note the following technical lemma, which belongs to the folklore.

Lemma 2.3. Let $N$ be an acyclic net, let $t$ be a transition, and let $\sigma$ be a minimal length firable sequence of transitions enabling $t$. Then every transition $x$ in $\sigma$ is such that $x<t$.

Proof. We first verify that if a marking $m$ enables a sequence of 2 transitions $t_{1} t_{2}$ such that $\neg\left(t_{1}<t_{2}\right)$, then $m$ also enables the sequence $t_{2} t_{1}$. Indeed, we have $m \geq \operatorname{Pre}\left(t_{1}\right)$ and $m-\operatorname{Pre}\left(t_{1}\right)+\operatorname{Post}\left(t_{1}\right) \geq$ $\operatorname{Pre}\left(t_{2}\right)$. Moreover, no place in the support of $\operatorname{Post}\left(t_{1}\right)$ is in the support of $\operatorname{Pre}\left(t_{2}\right)$ (otherwise $t_{1}<t_{2}$ ). Thus $m-\operatorname{Pre}\left(t_{1}\right) \geq \operatorname{Pre}\left(t_{2}\right)$, and this implies $m \geq \operatorname{Pre}\left(t_{2}\right)$ and $m-\operatorname{Pre}\left(t_{2}\right) \geq \operatorname{Pre}\left(t_{1}\right)$. The first inequality shows that $m$ enables $t_{2}$, and the second inequality shows that the marking obtained from $m$ by firing $t_{2}$ enables $t_{1}$.

Moreover, in this situation, the extended firing equation shows that the markings obtained after firing the sequences $t_{1} t_{2}$ and $t_{2} t_{1}$ coincide.

Now let $\sigma=t_{1} t_{2} \cdots t_{n}$ be a sequence of transitions such that $\sigma t$ is firable and $n$ is minimal. For each $1 \leq j \leq n$, let $m_{j}$ be the marking such that $m_{0}\left[t_{1} \cdots t_{j}\right\rangle m_{j}$. Thus $m_{i-1}$ enables $t_{i} t_{i+1} \cdots t_{n} t$ for each $i$. Now let $i$ be maximal such that $\neg\left(t_{i}<t\right)$. Then $t_{j}<t$ for each $j>i$, and hence $\neg\left(t_{i}<t_{j}\right)$. Using repeatedly the statement in the first part of the proof, it follows that $m_{i-1}$ enables $t_{i+1} t_{i} t_{i+2} \cdots t_{n} t$, and also $t_{i+1} t_{i+2} t_{i} \cdots t_{n} t$, etc, and finally $t_{i+1} \cdots t_{n} t t_{i}$, which contradicts the minimality of $n$.

\subsection{Homomorphism of nets}

Let $N=\left(P, T\right.$, Pre, Post, $\left.m_{0}\right)$ and $N^{\prime}=\left(P^{\prime}, T^{\prime}\right.$, Pre' ${ }^{\prime}$, Post $\left.{ }^{\prime}, m_{0}^{\prime}\right)$ be nets. Let $h: P \cup T \rightarrow P^{\prime} \cup T^{\prime}$ be a mapping such that $h(P) \subseteq P^{\prime}$ and $h(T) \subseteq T^{\prime}$. We say that $h$ is a homomorphism of nets from $N$ to $N^{\prime}$ (see [3]) if, for each transition $t \in T$, we have

- $\operatorname{Pre}^{\prime}(h(t))=h(\operatorname{Pre}(t))$,

- $\operatorname{Post}^{\prime}(h(t))=h(\operatorname{Post}(t))$,

- $m_{0}^{\prime}=h\left(m_{0}\right)$.

Observe that in this definition, $h\left(m_{0}\right), h(\operatorname{Pre}(t))$ and $h(\operatorname{Post}(t))$ must be well-defined, that is, the preimage $h^{-1}\left(p^{\prime}\right)$ of each place $p^{\prime} \in P^{\prime}$ must have a finite intersection with the support of $m_{0}$ and of each pre- and post-condition of a transition of $N$ (see Section 2.1).

We note the following elementary properties of homomorphisms of nets.

Lemma 2.4. Let $N=\left(P, T\right.$, Pre, Post, $\left.m_{0}\right)$ and $N^{\prime}=\left(P^{\prime}, T^{\prime}\right.$, Pre ${ }^{\prime}$, Post $\left.{ }^{\prime}, m_{0}^{\prime}\right)$ be nets, and let $h: N \rightarrow$ $N^{\prime}$ be a homomorphism.

1. Let $p^{\prime} \in P^{\prime}$. If $p^{\prime} \in \overline{m_{0}^{\prime}}$, then $h^{-1}\left(p^{\prime}\right)$ has at most $m_{0}^{\prime}\left(p^{\prime}\right)$ elements in $\overline{m_{0}}$. If $p^{\prime} \in \bullet h(t)$ for some transition $t \in T$, then $h^{-1}\left(p^{\prime}\right) \cap \bullet t$ has at most $\operatorname{Pre}^{\prime}\left(p^{\prime}, h(t)\right)$ elements. And if $p^{\prime} \in h(t)^{\bullet}$, then $h^{-1}\left(p^{\prime}\right) \cap t^{\bullet}$ has at most $\operatorname{Post}^{\prime}\left(p^{\prime}, h(t)\right)$ elements.

2. If $N$ has finitely many transitions, then $h^{-1}\left(p^{\prime}\right)$ contains a finite number of non-isolated or initially marked places for each place $p^{\prime} \in P^{\prime}$. 
3. If $m_{0}^{\prime}$ is safe, then $m_{0}$ is safe and $h$ induces a bijection from $\overline{m_{0}}$ to $\overline{m_{0}^{\prime}}$. Similarly, if $t \in T$ and $\operatorname{Pre}^{\prime}(h(t))$ (resp. Post $\left.t^{\prime}(h(t))\right)$ is a safe marking, then so is Pre $(t)$ (resp. Post $\left.(t)\right)$ and $h$ induces a bijection from $\bullet t$ to $\bullet h(t)$ (resp. from $t^{\bullet}$ to $h(t)^{\bullet}$ ).

4. If $N^{\prime}$ is weakly safe, then so is $N$.

Proof. To establish Statement (1), we first note that $m_{0}^{\prime}\left(p^{\prime}\right)=\sum_{h(p)=p^{\prime}} m_{0}(p)$, so $h^{-1}\left(p^{\prime}\right)$ contains at most $m_{0}^{\prime}\left(p^{\prime}\right)$ initially marked places. For each transition $t$ of $N, \operatorname{Pre}^{\prime}\left(p^{\prime}, h(t)\right)=\sum_{p=h\left(p^{\prime}\right)} \operatorname{Pre}(p, t)$, so $h^{-1}\left(p^{\prime}\right) \cap \bullet t$ has at most $\operatorname{Pre}^{\prime}\left(p^{\prime}, h(t)\right)$ elements. The reasoning is similar for the postset of $t$.

The other statements are simple consequences of (1).

Proposition 2.5. Let $N$ and $N^{\prime}$ be Petri nets and let $h: N \rightarrow N^{\prime}$ be a homomorphism. Let $m$ be a marking of $N$ such that $h(m)$ is well-defined, let $t$ be a transition of $N$ enabled by $m$, and let $m_{1}$ be the resulting marking, that is, $m[t\rangle m_{1}$. Then $h\left(m_{1}\right)$ is well-defined and $h(m)[h(t)\rangle h\left(m_{1}\right)$.

Proof. According to the firing equation, we have $m_{1}=m-\operatorname{Pre}(t)+\operatorname{Post}(t)$. The assumption that $h$ is a homomorphism implies that, for each place $p^{\prime}$ of $N^{\prime}$, the sums $\sum_{p \in h^{-1}\left(p^{\prime}\right)} \operatorname{Pre}(p, t)$ and $\sum_{p \in h^{-1}\left(p^{\prime}\right)} \operatorname{Post}(p, t)$ are well-defined. Similarly, stating that $h(m)$ is well-defined implies that each sum $\sum_{p \in h^{-1}\left(p^{\prime}\right)} m(p)$ is well-defined. It follows that

$$
h\left(m_{1}\right)\left(p^{\prime}\right)=\sum_{p \in h^{-1}\left(p^{\prime}\right)} m_{1}(p)=\sum_{p \in h^{-1}\left(p^{\prime}\right)}(m(p)-\operatorname{Pre}(p, t)+\operatorname{Post}(p, t))
$$

is well-defined, and is equal to

$$
h\left(m_{1}\right)\left(p^{\prime}\right)=h(m)\left(p^{\prime}\right)-\operatorname{Pre}^{\prime}\left(p^{\prime}, h(t)\right)+\operatorname{Post}^{\prime}\left(p^{\prime}, h(t)\right) .
$$

That is, $h(m)[h(t)\rangle h\left(m_{1}\right)$.

Proposition 2.5 can be extended by induction to sequences of transitions. This yields immediately the following corollary.

Corollary 2.6. Let $N$ and $N^{\prime}$ be Petri nets and let $h: N \rightarrow N^{\prime}$ be a homomorphism. The image under $h$ a reachable marking of $N$ is reachable in $N^{\prime}$. Moreover, if $N^{\prime}$ is safe, then $N$ is safe.

Example 2.7. Figure 2 shows both a net, say, $N$ (with 12 transitions and 13 places), and a homomorphism from this net to the net $N^{\prime}$ from Example 2.1. The values labelling the nodes are the images by the homomorphism. Thus, for instance, $N$ has 5 transitions which are mapped to the transition $b_{1}$ of $N^{\prime}$.

\subsection{Subnets of nets}

Let $N=\left(P, T\right.$, Pre, Post, $\left.m_{0}\right)$ and $N^{\prime}=\left(P^{\prime}, T^{\prime}\right.$, Pre $^{\prime}$, Post $\left.{ }^{\prime}, m_{0}^{\prime}\right)$ be nets. We say that $N^{\prime}$ is a subnet of $N$, and we write $N^{\prime} \sqsubseteq N$, if $P^{\prime} \subseteq P, T^{\prime} \subseteq T, m_{0}=m_{0}^{\prime}$ and for each transition $t$ of $N^{\prime}, \operatorname{Pre}(t)=\operatorname{Pre}^{\prime}(t)$ and $\operatorname{Post}(t)=\operatorname{Post}^{\prime}(t)$.

Observe that this definition is different from the sole requirement that $P^{\prime} \cup T^{\prime} \subseteq P \cup T$ and Pre', Post ${ }^{\prime}$ and $m_{0}^{\prime}$ are the restrictions of Pre, Post and $m_{0}$ to the places and transitions of $N^{\prime}$. Consider indeed 


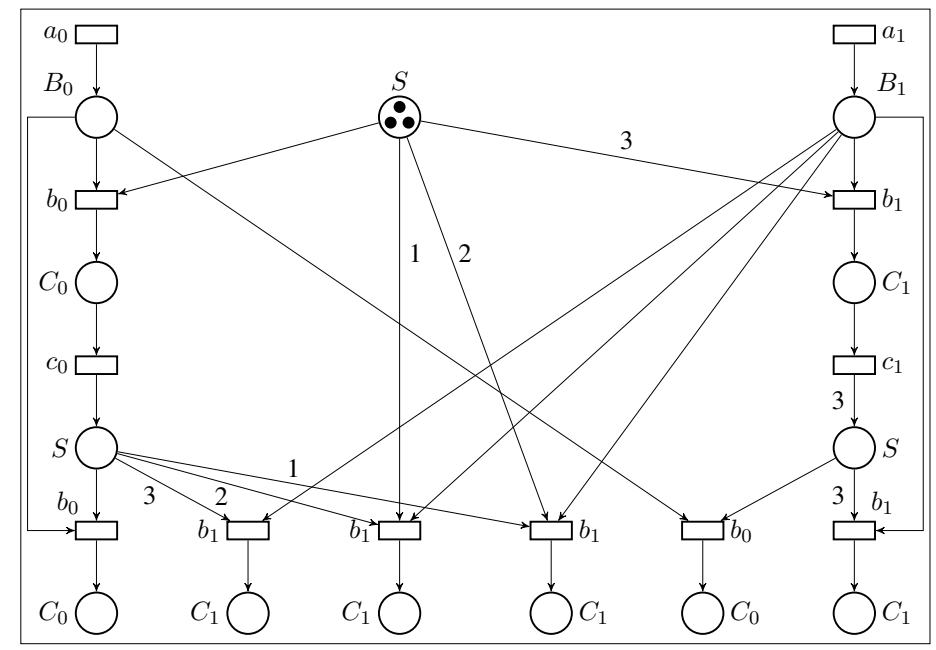

Figure 2. Graphical representation of a homomorphism of nets

the following situation: $N$ and $N^{\prime}$ have a single transition $t, N^{\prime}$ has places $p^{\prime}$ and $q^{\prime}$, and $N$ has places $p, q, p^{\prime}$ and $q^{\prime}, m_{0}=m_{0}^{\prime}=p^{\prime}, \operatorname{Pre}^{\prime}(t)=p^{\prime}, \operatorname{Pre}(t)=p+p^{\prime}, \operatorname{Post}^{\prime}(t)=q^{\prime}, \operatorname{Post}(t)=q+q^{\prime}$. The containment and restriction conditions above hold, yet $N^{\prime}$ is not a subnet of $N$.

In our definition, a subnet of $N$ is obtained by removing from $N$ some places and transitions, in a way that impacts neither the initial marking, nor the pre- and post-conditions of the remaining transitions. In other words, a subnet is uniquely determined by a set of transitions, the initial marking and, possibly, a set of isolated, non-initially marked places.

\section{Occurrence nets and configurations of a net}

A net $N$ is an occurrence net if, for each place $p \in P$,

- either $p$ is initial $\left({ }^{\bullet} p=\emptyset\right)$ or it receives its inputs from a single transition $\left(\left.\right|^{\bullet} p \mid=1\right)$, denoted by $\cdot p$

- the support of $m_{0}$ is exactly the set of initial places $\left(m_{0}(p)>0\right.$ if and only if $p$ is an initial place);

- $N$ is quasi-live (every transition is enabled by some reachable marking).

Occurrence nets were structurally introduced by Nielsen, Plotkin and Winskel [13] in the safe case using concurrency relation over transitions and places. Their definition differs from ours by the fact that it imposes acyclicity and the absence of self-conflicts. We have retained natural structural constraints such as the fact that a place is either initial and initially marked, or unmarked and in the postset of a single transition. Self-conflict is more difficult to handle in a structural way in the setting of general nets and we have introduced the condition of quasi-liveness in the definition in order to "trim" unnecessary transitions. As we will see, acyclicity follows from our definition. Our definition remains simple, and it makes no assumption of safeness or finiteness. It is important to note that the two definitions coincide for safe nets (see subsection 3.1). 


\subsection{Elementary properties of occurrence nets}

Proposition 3.1. Let $N=\left(P, T\right.$, Pre, Post, $\left.m_{0}\right)$ be an occurrence net. Then $N$ is acyclic and every vertex of the graph $N$ is preceded by a finite number of transitions. That is, if $x \in P \cup T$, then ${ }^{*} x \cap T$ is finite.

Proof. As the graph (underlying) $N$ is bipartite, if $N$ has a cycle, then there exists a sequence of places $p_{0}, p_{1}, \ldots, p_{n-1}$ and a sequence of transitions $t_{0}, t_{1}, \ldots, t_{n-1}$ such that ${ }^{\bullet} p_{i}=t_{i}$ and $t_{i} \in p_{i-1}$ for $i=0, \ldots, n$ (where $i$ is taken modulo $n$ ). The value at $p_{i}$ of a marking of $N$ can be modified only by firing transition $t_{i}$, and transition $t_{i}$ is enabled by a marking $m$ only if $m\left(p_{i-1}\right) \neq 0$. Finally, as none of the places $p_{i}$ is initial, the initial marking $m_{0}$ is 0 on each $p_{i}$ : it is now immediately verified, by induction on the length of a firing sequence, that no reachable marking enables any of the transitions $t_{i}$, thus contradicting the assumption that $N$ is quasi-live. $\stackrel{*}{\rightarrow}$.

Thus $N$ is acyclic. In particular, the set of vertices of the graph $N$ is partially ordered by the relation

Let $p \in P$ be a place that is not initial: then ${ }^{*} p \cap T={ }^{*}(\bullet p) \cap T$ by definition of an occurrence net. Thus it suffices to show that ${ }^{*} t \cap T$ is finite for every transition $t$.

Observe that if $t^{\prime} \in T$ and $q \in P$ are such that $t^{\prime} \rightarrow q \rightarrow t$ in $N$, then $t^{\prime}={ }^{\bullet} q$ and $t$ can be fired only after $t^{\prime}$ was fired. This remark is extended by induction to show that if $t^{\prime}$ is a transition and $t^{\prime} \stackrel{t}{\rightarrow} t$, then $t^{\prime}$ must be fired before $t$ can be fired. As an occurrence net is quasi-live, that is, every transition can appear in a finite sequence of transitions enabled by the initial marking, it follows that the set of transitions in * $t$ is finite.

We note the following property of subnets of occurrence nets. A subnet $N^{\prime}$ of an acyclic net $N$ is a prefix of $N$ if $P^{\prime} \cup T^{\prime}$ is an order ideal of $P \cup T$.

Lemma 3.2. Let $N$ be an occurrence net and let $N^{\prime}$ be a subnet of $N$. Then $N^{\prime}$ is an occurrence net if and only if $N^{\prime}$ is a prefix of $N$.

Proof. It is immediate that a prefix of an occurrence net is an occurrence net. Conversely, suppose that $N^{\prime} \sqsubseteq N$. Then $\operatorname{Pre}(t)=\operatorname{Pre}^{\prime}(t)$ for each transition $t$ of $N^{\prime}$. In particular, if $t$ is a transition of $N^{\prime}$ and $p \rightarrow t$ in $N$, then $p \in P^{\prime}$.

If in addition $N^{\prime}$ is an occurrence net, consider a place $p$ of $N^{\prime}$. If $t \rightarrow p$ in $N$, then $m_{0}(p)=0$. If $t$ is not in $N^{\prime}$, then ${ }^{\bullet} p=\emptyset$ in $N^{\prime}$, so that $m_{0}^{\prime}(p)>0$, contradicting the equality $m_{0}=m_{0}^{\prime}$. Thus $t$ is in $N^{\prime}$ and $N^{\prime}$ is a prefix of $N$.

For occurrence nets without spontaneous, non-isolated transitions, the distinction between safeness and weak safeness vanishes (see Lemma 2.2). Note that this includes the weakly safe occurrence nets in Engelfriet's definition [3].

Proposition 3.3. Let $N$ be an occurrence net without spontaneous, non-isolated transitions. Then $N$ is safe if and only if $N$ is weakly safe. Moreover, in a safe occurrence net, a non-spontaneous transition may occur at most once in a firing sequence enabled by the initial marking. Finally, in a safe occurrence net, there can be no self-conflict: that is, if $t, t_{1}, t_{2}$ are transitions with $t_{1} \stackrel{*}{\rightarrow} t$ and $t_{2} \stackrel{*}{\rightarrow} t$, then $t_{1}=t_{2}$ or ${ }^{\bullet} t_{1} \cap t_{2}$ is empty. 
Proof. In view of Lemma 2.2, it suffices to show that if $N$ is weakly safe, then it is safe. We consider a marking $m$ of $N$, reached after firing a sequence of transitions $\sigma=t_{1} t_{2} \ldots t_{n}$ from the initial marking $m_{0}$, and we denote by $m_{i}$ the marking reached after firing $t_{1} \cdots t_{i}$.

We show that for each place $p$, either $m_{k}(p)=0$ for each $0 \leq k \leq n$, or there exists $0 \leq i \leq j \leq n$ such that $m_{k}(p)=0$ for $k<i$ or $k>j$ and $m_{k}(p)=1$ for $i \leq k \leq j$. This shows that $m$ is a safe marking, and hence that $N$ is safe. Moreover, it shows that if $t$ is not a spontaneous transition, then $t$ occurs at most once in $\sigma$ (by considering a place $p \in \bullet$ ). It also shows that, if $\bullet_{1} \cap t_{2}$ is not empty, then $t_{1}$ and $t_{2}$ may not occur in the same firing sequence. In particular, the quasi-liveness condition implies that there is no transition $t$ such that $t_{1} \stackrel{*}{\rightarrow} t$ and $t_{2} \stackrel{*}{\rightarrow} t$.

The proof is by induction on $\left.\right|^{*} p \cap T \mid$, which is finite by Proposition 3.1.

If $\left.\right|^{*} p \cap T \mid=0$, then $p$ is an initial place, so $m_{0}(p)=1$ (since $m_{0}$ is safe). If the marking of $p$ is not constantly 1 , let $j$ be minimal such that $m_{j+1}(p) \neq 1$. Then $m_{k}(p)=1$ for $k \leq j$, $\operatorname{Pre}\left(p, t_{j+1}\right) \neq 0$ and $m_{j+1}(p)=0$. Since $p$ is initial, the marking of $p$ remains 0 for the rest of the sequence.

Now assume that $\ell \geq 1$ and the property holds for all places in whose past there are at most $\ell-1$ transitions. Let $p$ be such that $\left.\right|^{*} p \cap T \mid=\ell$. Since $p$ is not initial, we have $m_{0}(p)=0$. If the marking of $p$ is not constantly 0 , let $i$ be minimal such that $m_{i}(p) \neq 0$. Then $t_{i}={ }^{\bullet} p$ and $m_{i}(p)=\operatorname{Post}\left(p, t_{i}\right)=1$ since $N$ is weakly safe. Since $t_{i}$ is not isolated, it is also not spontaneous and we consider a place $q \in{ }^{\bullet} t_{i}={ }^{\bullet} p$. Since $N$ is acyclic (Proposition 3.1), $t_{i} \notin{ }^{*} q \subset{ }^{*} p$, so by induction, we find that $m_{i-1}(q)=1$ (since $t_{i}$ is enabled after firing $\left.t_{1} \cdots t_{i-1}\right), m_{i}(q)=0$ and $m_{k}(q)=0$ for all $k \geq i$. Therefore $t_{i}$ will never be enabled again (and hence $t_{i} \neq t_{k}$ for $k>i$ ) and $m_{k}(p) \leq 1$ for all $k \geq i$. Further, if the marking of $p$ does not remain 1 for the rest of the sequence, let $j$ be minimal such that $j \geq i$ and $m_{j+1}(p) \neq 1$. This means that $\operatorname{Pre}\left(p, t_{j}\right) \neq 0$ and $m_{j+1}(p)=0$ (by acyclicity). And since $t_{i}=\bullet p$ will not be fired again, we have $m_{k}(p)=0$ for all $k>j$.

\subsection{Configurations}

Let $N$ be a Petri net. The multiset of transitions induced by a finite firable sequence of transitions is called a configuration of $N$.

It is not true that, even in an occurrence net, a configuration arises from a unique firable sequence: suppose $P=\left\{p_{1}, \ldots, p_{4}\right\}, T=\left\{t_{1}, t_{2}\right\}, m_{0}=p_{1}+p_{2}$, Pre $=\left(p_{1}, t_{1}\right)+\left(p_{2}, t_{2}\right)$ and Post $=$ $\left(p_{3}, t_{1}\right)+\left(p_{4}, t_{2}\right)$. Then $t_{1} t_{2}$ and $t_{2} t_{1}$ are distinct firable sequences yielding the same configuration. More generally, transitions with disjoint pre-conditions can be fired in any order.

It follows however immediately from the extended firing equation that if two firable sequences induce the same configuration $\varphi$, then they both lead to the same marking, denoted by $\operatorname{Cut}(\varphi)$. Somewhat abusing definitions, we say that $\operatorname{Cut}(\varphi)$ is the marking reached after firing the vector (or the configuration) $\varphi$.

Configurations of acyclic nets are characterized as follows ([12] for the case of finite nets).

Proposition 3.4. Let $N$ be a Petri net and let $\varphi$ be a multiset over $T$. If $\varphi$ is a configuration, then the support of $\varphi$ is finite and, for each place $p \in P$, we have

$$
m_{0}(p)-\sum_{t \in T} \operatorname{Pre}(p, t) \cdot \varphi(t)+\sum_{t \in T} \operatorname{Post}(p, t) \cdot \varphi(t) \geq 0
$$

If $N$ is acyclic and $\varphi$ is a finite support multiset over $T$ satisfying Equation (1) for all places $p$, then $\varphi$ is a configuration. 
Proof. If $\varphi$ is a configuration of the Petri net $N$, then the support of $\varphi$ is trivially finite, and for each place $p, \operatorname{Cut}(\varphi)(p) \geq 0$ : that is exactly the statement in Equation (1).

We now assume that $N$ is acyclic, $\varphi$ has finite support and Equation (1) holds for each place $p$. We proceed by induction on the value of $\sum_{t \in T} \varphi(t)$. The empty multiset is certainly a configuration, since it is the sum of the terms of the empty sequence of transitions. Now we assume that $\varphi \neq 0$. The relation $\stackrel{*}{\rightarrow}$ is a partial order by assumption, and we consider a transition $s$ maximal in the support of $\varphi$ and the multiset $\boldsymbol{\psi}$ such that $\boldsymbol{\psi}+s=\boldsymbol{\varphi}$. Of course, $\boldsymbol{\psi}$ has finite support.

Let $m_{\boldsymbol{\psi}}(p)=m_{0}(p)-\sum_{t \in T} \operatorname{Pre}(p, t) \cdot \boldsymbol{\psi}(t)+\sum_{t \in T} \operatorname{Post}(p, t) \cdot \boldsymbol{\psi}(t)$. Then Equation (1) states that

$$
m_{\psi}(p)-\operatorname{Pre}(p, s)+\operatorname{Post}(p, s) \geq 0 .
$$

We want to show that $m_{\boldsymbol{\psi}}(p) \geq 0$ for each place $p$. If $\operatorname{Post}(p, s)=0$, then $m_{\boldsymbol{\psi}}(p) \geq \operatorname{Pre}(p, s) \geq 0$. If $\operatorname{Post}(p, s) \neq 0$, then $s \in \in^{\bullet} p$, and since $s$ is maximal in the support of $\varphi, \operatorname{Pre}(p, t)=0$ for each transition $t$ in the support of $\varphi$. In particular,

$$
m_{\boldsymbol{\psi}}(p)=m_{0}(p)+\sum_{t \in T} \operatorname{Post}(p, t) \cdot \boldsymbol{\psi}(t) \geq m_{0}(p) \geq 0 .
$$

We can now use the induction hypothesis to see that $\psi$ is a configuration - and hence, $m_{\psi}=\operatorname{Cut}(\boldsymbol{\psi})$. We already noticed that if $\operatorname{Post}(p, s)=0$, then $m_{\psi}(p) \geq \operatorname{Pre}(p, s)$. Moreover, if $\operatorname{Post}(p, s) \neq 0$, then $\operatorname{Pre}(p, s)=0$ by acyclicity and again, we have $m_{\psi}(p) \geq \operatorname{Pre}(p, s)$. Thus the transition $s$ is enabled by the marking $\operatorname{Cut}(\psi)$ and $\boldsymbol{\varphi}=\boldsymbol{\psi}+s$ is a configuration.

In view of the specific properties of occurrence nets, Proposition 3.4 yields the following corollary.

Corollary 3.5. Let $N$ be an occurrence net and let $\varphi$ be a multiset over $T$. Then $\varphi$ is a configuration of $N$ if and only if $\varphi$ has finite support and for each place $p \in P$, we have

$$
\begin{aligned}
& m_{0}(p) \geq \sum_{t \in T} \operatorname{Pre}(p, t) \cdot \boldsymbol{\varphi}(t) \quad \text { if } p \text { is an initial place, } \\
& \operatorname{Post}\left(p,{ }^{\bullet} p\right) \cdot \boldsymbol{\varphi}\left({ }^{\bullet} p\right) \quad \geq \sum_{t \in T} \operatorname{Pre}(p, t) \cdot \boldsymbol{\varphi}(t) \quad \text { otherwise. }
\end{aligned}
$$

We also verify that, as in the safe case, under a mild assumption, each reachable marking of an occurrence net is reached after firing a uniquely determined configuration - thus generalizing a property of occurrence nets in the classical setting.

Proposition 3.6. Let $N$ be an occurrence net such that $t^{\bullet} \neq \emptyset$ for each transition $t$, and let $m$ be a reachable marking. Then there exists a unique configuration $\varphi$ of $N$ such that $m=\operatorname{Cut}(\varphi)$.

Proof. Since $m$ is a reachable marking, it is reached after firing a sequence $\sigma$, and $m=\operatorname{Cut}(\varphi)$, where $\varphi$ is the configuration induced by $\sigma$. We denote by $|\varphi|$ the cardinality of the support of $\varphi$.

We want to show that $\operatorname{Cut}(\varphi)=\operatorname{Cut}\left(\varphi^{\prime}\right)$ implies $\varphi=\varphi^{\prime}$. Without loss of generality, we may assume that $|\varphi| \geq\left|\varphi^{\prime}\right|$. We proceed by induction on $|\varphi|$. If $|\varphi|=0$, then $\varphi$ and $\varphi^{\prime}$ are both equal to the empty configuration and the equality $\varphi=\varphi^{\prime}$ holds.

Let us now consider configurations $\varphi$ and $\varphi^{\prime} \operatorname{such}$ that $\varphi$ is not empty and $m=\operatorname{Cut}(\varphi)=\operatorname{Cut}\left(\varphi^{\prime}\right)$. Let $s$ be a maximal transition in the support of $\varphi$ (using the acyclicity of occurrence nets). Then the places in $s^{\bullet}$ are in the support of $m$, and as these places may receive tokens only from $s, s$ must be also 
in the multiset $\varphi^{\prime}$. For the same reason, $s$ is maximal in the support of $\varphi^{\prime}$. Moreover, for each place $p \in s^{\bullet}, m(p)=\varphi(s)=\varphi^{\prime}(s)$, so $\varphi$ and $\varphi^{\prime}$ contain the same number of occurrences of $s$.

Let $\psi$ and $\psi^{\prime}$ be the multisets obtained from $\varphi$ and $\varphi^{\prime}$ by removing all occurrences of $s$. Corollary 3.5 shows that $\psi$ and $\psi^{\prime}$ are configurations as well: indeed, in the first inequality, the left-hand side remains constant and the right-hand side may only decrease when going from $\varphi$ to $\psi$ (resp. from $\varphi^{\prime}$ to $\psi^{\prime}$ ); the same situation holds in the second inequality, except for the places $p \in s^{\bullet}$, but for these places, the right-hand side is zero since $s$ is maximal and $N$ is acyclic.

The maximality of $s$ in the support of $\varphi$ implies that any firing sequence $\sigma$ which induces the configuration $\varphi$ can be rearranged in the form $\sigma_{1} \sigma_{2}$ where $\sigma_{2}$ consists of $\varphi(s)$ occurrences of $s$. In particular, the configuration induced by $\sigma_{1}$ is $\psi$ and it follows from the extended firing equation that

$$
\operatorname{Cut}(\psi)=m+\operatorname{Pre}(s) \cdot \boldsymbol{\varphi}(s)-\operatorname{Post}(s) \cdot \boldsymbol{\varphi}(s) .
$$

Similarly, $\operatorname{Cut}\left(\psi^{\prime}\right)=m+\operatorname{Pre}(s) \cdot \boldsymbol{\varphi}^{\prime}(s)-\operatorname{Post}(s) \cdot \boldsymbol{\varphi}^{\prime}(s)$ and hence, $\operatorname{Cut}(\boldsymbol{\psi})=\operatorname{Cut}\left(\boldsymbol{\psi}^{\prime}\right)$. We conclude by induction that $\psi=\psi^{\prime}$, and since $\varphi(s)=\varphi^{\prime}(s)$, we have $\varphi=\varphi^{\prime}$.

Remark 3.7. The same uniqueness result holds (with essentially the same proof) for any acyclic net such that $t^{\bullet} \neq \emptyset$ for each transition $t$ and $\left|{ }^{\bullet} p\right| \leq 1$ for each place $p$.

\section{Branching processes and unfoldings of a net}

We now discuss the branching processes and the unfoldings of a net [3], within the framework developped in this paper. As with occurrence nets, we need to revisit the classical definition of a branching process: our definition makes no reference to the notion of conflict, and accounts for the fact that a condition may receive several tokens and an event may occur several times. Bowing to tradition, occurrence nets in branching processes will be usually written $S=\left(B, E, \ln\right.$, Out, $\left.q_{0}\right)$, their transitions will be called events and their places will be called conditions.

\subsection{Branching processes}

A branching process of a net $N$ is a pair $(S, h)$ consisting of an occurrence net $S=\left(B, E, \ln\right.$, Out, $\left.q_{0}\right)$ and a homomorphism $h: S \rightarrow N$ satisfying a guarded form of injectivity: whenever $e$ and $e^{\prime}$ are events of $S$,

$$
\text { if } \ln (e)=\ln \left(e^{\prime}\right) \text { and } h(e)=h\left(e^{\prime}\right) \text { then } e=e^{\prime} .
$$

The branching process $(S, h)$ is called safe (resp. weakly safe) if $S$ is safe (resp. weakly safe). Note that a weakly safe branching process without spontaneous, non-isolated events is safe (see Proposition 3.3). We also note the following fact (see Lemma 2.4 (4) and Corollary 2.6).

Proposition 4.1. If $N$ is safe (resp. weakly safe), then every branching process of $N$ is safe (resp. weakly safe) as well.

We now introduce a new property of branching processes.

Definition 4.2. We say that a branching process $(S, h)$ is faithful if $h$ is injective on $\overline{q_{0}}$, the support of the initial marking of $S$, and on the post-set $e^{\bullet}$ of each event $e$ of $S$. 


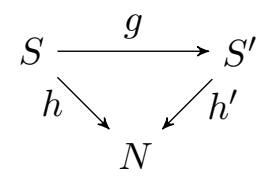

Figure 3. $g:(S, h) \rightarrow\left(S^{\prime}, h^{\prime}\right)$ is a homomorphism of branching processes

Example 4.3. The net presented in Figure 2 is an occurrence net. With the homomorphism shown in the figure, it constitutes a faithful branching process of the net from Example 2.1.

Let $(S, h)$ and $\left(S^{\prime}, h^{\prime}\right)$ be branching processes of $N$ and let $g: S \rightarrow S^{\prime}$ be a homomorphism of nets. We say that $g$ is a homomorphism of branching processes if $h=h^{\prime} \circ g$, see Figure 3, and we write $g:(S, h) \rightarrow\left(S^{\prime}, h^{\prime}\right)$. We will use the following technical properties of homomorphisms of branching processes.

Lemma 4.4. Let $S$ and $S^{\prime}$ be occurrence nets, $h: S \rightarrow N, h^{\prime}: S^{\prime} \rightarrow N$ and $g: S \rightarrow S^{\prime}$ be homomorphisms such that $h=h^{\prime} \circ g$ and assume that $\left(S^{\prime}, h^{\prime}\right)$ is a branching process of $N$.

1. $(S, h)$ is a branching process of $N$ if and only if $(S, g)$ is a branching process of $S^{\prime}$.

2. If $\left(S^{\prime}, h^{\prime}\right)$ is faithful, then $(S, h)$ is a faithful branching process of $N$ if and only if $(S, g)$ is a faithful branching process of $S^{\prime}$.

3. If $\left(S^{\prime}, h^{\prime}\right)$ is faithful, then $g$ is the only net homomorphism from $S$ to $S^{\prime}$ such that $h^{\prime} \circ g=h$.

4. If $(S, h)$ and $\left(S^{\prime}, h^{\prime}\right)$ are faithful branching processes, then $g$ is injective.

Proof. Let $S=\left(B, E, \ln\right.$, Out, $\left.q_{0}\right), S^{\prime}=\left(B^{\prime}, E^{\prime}, \operatorname{In}^{\prime}\right.$, Out $\left.{ }^{\prime}, q_{0}^{\prime}\right)$ and let $e, e^{\prime}$ be events of $S$ such that $\ln (e)=\ln \left(e^{\prime}\right)$. We first assume that $(S, h)$ is a branching process of $N$ and that $g(e)=g\left(e^{\prime}\right)$. Then $h(e)=h^{\prime}(g(e))$ and $h\left(e^{\prime}\right)=h^{\prime}\left(g\left(e^{\prime}\right)\right)$ are equal: as $(S, h)$ is a branching process, it follows that $e=e^{\prime}$. Thus $(S, g)$ is a branching process.

Conversely, suppose that $(S, g)$ is a branching process and $h(e)=h\left(e^{\prime}\right)$. Since $g$ is a homomorphism, we have $\ln ^{\prime}(g(e))=g(\ln (e))$ and $\ln ^{\prime}\left(g\left(e^{\prime}\right)\right)=g\left(\ln \left(e^{\prime}\right)\right)$, so that $\ln ^{\prime}(g(e))=\ln ^{\prime}\left(g\left(e^{\prime}\right)\right)$. Moreover, $h^{\prime}(g(e))=h(e)=h\left(e^{\prime}\right)=h^{\prime}\left(g\left(e^{\prime}\right)\right)$ : since $\left(S^{\prime}, h^{\prime}\right)$ is a branching process, we have $g(e)=g\left(e^{\prime}\right)$, and since $(S, g)$ is a branching process as well, we have $e=e^{\prime}$, which concludes the proof of Property (1).

The verification of the preservation of faithful branching processes, that is, of Property (2), is analogous.

We now turn to Property (3) and we assume that $\left(S^{\prime}, h^{\prime}\right)$ is faithful. Let $g^{\prime}: S \rightarrow S^{\prime}$ be a homomorphism of nets such that $h^{\prime} \circ g^{\prime}=h$. If $b \in \overline{q_{0}}$, then $h(b)=h^{\prime}(g(b))=h^{\prime}\left(g^{\prime}(b)\right)$ with $g(b), g^{\prime}(b) \in \overline{q_{0}^{\prime}}$, and since $h^{\prime}$ is injective on $\overline{q_{0}^{\prime}}$, we have $g(b)=g^{\prime}(b)$. Thus $g$ and $g^{\prime}$ coincide on $\overline{q_{0}}$, a prefix of $S$. Let $I$ be a maximal prefix of $S$ on which $g$ and $g^{\prime}$ coincide (the existence of such a prefix is ensured either by an application of Zorn's lemma) and let $e$ be an event of $S \backslash I$. Since $e$ has finitely many transitions in its past (Proposition 3.1), we may choose $e$ such that ${ }^{\bullet} e \subseteq I$. Then $\ln ^{\prime}(g(e))=g(\ln (e))=g^{\prime}(\operatorname{In}(e))=$ $\operatorname{In}^{\prime}\left(g^{\prime}(e)\right)$. We also have $h^{\prime}(g(e))=h(e)=h^{\prime}\left(g^{\prime}(e)\right)$, and hence $g(e)=g^{\prime}(e)$ since $\left(S^{\prime}, h^{\prime}\right)$ is a branching process. Moreover, if $b \in e^{\bullet}$, then $h^{\prime}(g(b))=h(b)=h^{\prime}\left(g^{\prime}(b)\right)$, and since $h^{\prime}$ is injective on $g(e)^{\bullet}$, 
we have $g(b)=g^{\prime}(b)$. Thus $g$ and $g^{\prime}$ coincide on the prefix $I \cup\{e\} \cup e^{\bullet}$ of $S$, a contradiction. Therefore $I=S$, that is, $g=g^{\prime}$.

Finally, we verify Property (4), assuming that both $(S, h)$ and $\left(S^{\prime}, h^{\prime}\right)$ are faithful branching processes. Let $b_{1} \in \overline{q_{0}}$. Then $g\left(b_{1}\right) \in \overline{q_{0}^{\prime}}$. If $b_{2}$ is a condition of $S$ such that $g\left(b_{2}\right)=g\left(b_{1}\right)$, then $b_{2} \in \overline{q_{0}}$ : if it is not the case, then $S$ has an event $e=\bullet b_{2}$ and $g\left(b_{1}\right)=g\left(b_{2}\right) \in g(e)^{\bullet}$, a contradiction. By Property (2), $(S, g)$ is faithful, so $g$ is injective on $\overline{q_{0}}$ and hence $b_{1}=b_{2}$.

As in the verification of Property (3), let $I$ be a maximal prefix of $S$ such that $g^{-1}(g(x))$ for every $x \in I$. Let $e$ be an event of $S \backslash I$, which we may choose such that ${ }^{\bullet} e \subseteq I$. Then there exists an event $e^{\prime}$ such that $g(e)=g\left(e^{\prime}\right)$. Since $\ln ^{\prime}(g(e))=g(\ln (e))=g\left(\ln \left(e^{\prime}\right)\right)$, the hypothesis on $I$ implies that $\ln (e)=\ln \left(e^{\prime}\right)$. Since $(S, g)$ is a branching process of $S^{\prime}$ (by Property (1)), it follows that $e=e^{\prime}$.

Moreover, let $b \in e^{\bullet}$ and let $b^{\prime}$ be a condition such that $g(b)=g\left(b^{\prime}\right)$. Then $b^{\prime} \notin \overline{q_{0}^{\prime}}$ (by the argument developed above). In particular, we have $g\left(\bullet b^{\prime}\right)=g(\bullet b)=g(e)$ and, as we just saw, it follows that $\bullet b^{\prime}=e$. But $g$ is injective on $e^{\bullet}$, which contains both $b$ and $b^{\prime}$, so $b=b^{\prime}$. The consideration of the prefix $I \cup\{e\} \cup e^{\bullet}$ contradicts the maximality of $I$, and therefore $g$ is injective on $S$.

\subsection{Unfoldings of a net}

We extend the natural partial order on the subnets of a net to branching processes to the following quasiorder (see [3]): we say that $(S, h) \preceq\left(S^{\prime}, h^{\prime}\right)$ if there exists an injective homomorphism of branching processes from $(S, h)$ to $\left(S^{\prime}, h^{\prime}\right)$. In view of Lemma 3.2, this is equivalent to stating that $(S, h)$ is isomorphic to a prefix of $\left(S^{\prime}, h^{\prime}\right)$.

A $\preceq$-maximal branching process of $N$ is called an unfolding of $N$. The following characterization of unfoldings plays a major rôle in the sequel. We say that a marking $v$ of a net is covered if there exists a reachable marking $m$ such that $v \leq m$.

Proposition 4.5. A branching process $(S, h)$ of a net $N$ is an unfolding if and only if the following property holds:

$(\dagger)$ if $t$ is a transition of $N$ such that $\operatorname{Pre}(t)=h(v)$ for some marking $v$ covered in $S$, then there exists an event $e$ of $S$ such that $h(e)=t$ and $\ln (e)=v$.

Proof. Let us first assume that $(S, h)$ satisfies Property $(\dagger)$ and let $\left(S^{\prime}, h^{\prime}\right)$ be a branching processes of $N$ such that $(S, h) \preceq\left(S^{\prime}, h^{\prime}\right)$. We want to show that $(S, h)$ and $\left(S^{\prime}, h^{\prime}\right)$ are isomorphic. Without loss of generality, we may assume that $(S, h)$ is a prefix of $\left(S^{\prime}, h^{\prime}\right)$, that is, $S \sqsubseteq S^{\prime}$, and we show that $S=S^{\prime}$.

We first verify that if $S$ and $S^{\prime}$ contain the same events, then they also contain the same conditions, and hence they are equal. Indeed, the assumption that $S \sqsubseteq S^{\prime}$ implies that $S$ and $S^{\prime}$ have the same initial conditions and that, for each event $e$ of $S, S$ contains all the conditions of $S^{\prime}$ which are in ${ }^{\bullet} e$ or $e^{\bullet}$; finally the definition of occurrence nets implies that all the conditions of $S^{\prime}$ are either initial or in the postset of an event.

Thus if $S \neq S^{\prime}$, there is an event $e^{\prime}$ of $S^{\prime}$ not in $S$. In the acyclic net $S^{\prime}$, we can choose $e^{\prime}$ to be $\leq$-minimal (by Proposition 3.1).

Since $S^{\prime}$ is quasi-live, there exists a firable sequence of events $\sigma$ such that the sequence $\sigma e^{\prime}$ is firable; in addition, $\sigma$ can be assumed to consist only of events that are $\leq-$ less than $e^{\prime}$ (by Lemma 2.3). By minimality of $e^{\prime}, \sigma$ is also a firable sequence of $S$ and in particular, the conditions in $\ln ^{\prime}\left(e^{\prime}\right)$ are in $S$. If $v$ is the marking reached after the firing of $\sigma$ in $S^{\prime}, m_{0}^{\prime}[\sigma\rangle v$, then the support of $v$ is in $S$ by definition of subnets, so that $m_{0}[\sigma\rangle v$ in $S$ as well. 
Now, Pre $\left(h^{\prime}\left(e^{\prime}\right)\right)=h^{\prime}\left(\operatorname{In}^{\prime}\left(e^{\prime}\right)\right)$ and $\operatorname{In}^{\prime}\left(e^{\prime}\right)$ is covered by the marking $v$, which is reachable in $S$. By Property (†), there exists an event $e$ of $S$ such that $h(e)=h^{\prime}\left(e^{\prime}\right)$ and $\ln (e)=\ln ^{\prime}\left(e^{\prime}\right)$. But $h$ is a restriction of $h^{\prime}$, so $h^{\prime}(e)=h^{\prime}\left(e^{\prime}\right)$ and, by definition of a branching process, we get $e=e^{\prime}$, a contradiction. Thus, if $(S, h)$ satisfies Property $(\dagger)$, then $(S, h)$ is $\preceq$-maximal among the branching processes of $N$, that is, $(S, h)$ is an unfolding of $N$.

Conversely, let us assume that $(S, h)$ does not satisfy Property $(\dagger)$, that is, there exists a transition $t$ of $N$ which does not lie in $h(S)$, such that $\operatorname{Pre}(t)=h(v)$ for some marking $v$ of $S$, which is covered in $S$. Then there exists a firable sequence of $S, \sigma$, such that $v \leq \operatorname{Cut}(\sigma)$. Let us construct a new net $S^{\prime}$ by adding to $S$ one new event, say $e$, and a set $Q$ of new conditions, equipped with a bijection $\theta$ onto the support of $\operatorname{Post}(t)$. We let $\operatorname{Out}^{\prime}(e)=\sum_{q \in Q} \operatorname{Post}(\theta(q), t) q, \operatorname{In}^{\prime}(e)=v$, and for each event $f$ of $S$, $\operatorname{In}^{\prime}(f)=\ln (f)$ and $\operatorname{Out}^{\prime}(f)=\operatorname{Out}(f)$. We also consider the extension $h^{\prime}$ of the homomorphism $h$ given by $h^{\prime}(e)=t$ and $h^{\prime}(q)=\theta(q)$ for each $q \in Q$. In order to conclude, we need to verify that $\left(S^{\prime}, h^{\prime}\right)$ is a branching process of $N$ and $h$ is the restriction of $h^{\prime}$ to $S$, which is immediate. This shows that $(S, h)$ is not $\preceq$-maximal, and hence not an unfolding.

We note the following applications of Proposition 4.5, which express important properties of unfoldings. The first one states that unfoldings simulate all the firable sequences of a net, the second one deals with homomorphisms between unfoldings, and the last one considers unfoldings of unfoldings.

Corollary 4.6. Let $(S, h)$ be an unfolding of the net $N$. If $\sigma$ is a firable sequence of $N$, then there exists a firable sequence $\rho$ of $S$ such that $h(\rho)=\sigma$.

Proof. Let $N=\left(P, T\right.$, Pre, Post, $\left.m_{0}\right)$ and $S=\left(B, E\right.$, In, Out, $\left.q_{0}\right)$. We first observe the following elementary fact: let $m$ be a multiset over $P$ and $v$ be a multiset over $B$ such that $m \leq h(v)$. Then there exists a multiset $v^{\prime}$ over $B$ such that $m=h\left(v^{\prime}\right)$ and $v^{\prime} \leq v$. Indeed, for each $p \in P$, we have $m(p) \leq h(v)(p)=\sum_{b \in B, h(b)=p} v(b)$ : one can choose, for each $b \in B$ such that $h(b)=p$, a value $0 \leq v^{\prime}(b) \leq v(p)$ such that $m(p)=\sum_{b \in B, h(b)=p} v^{\prime}(b)$.

We now proceed by induction on the length $n$ of $\sigma$. If $n=0$, the statement is trivially true. We now assume that $\sigma=\sigma^{\prime} t$ and we let $w$ be the marking such that $m_{0}\left[\sigma^{\prime}\right\rangle w$. Since $\sigma$ is firable, $\operatorname{Pre}(t) \leq w$. And by induction hypothesis, there exists a firable sequence $\rho^{\prime}$ of $S$ such that $h\left(\rho^{\prime}\right)=\sigma^{\prime}$. In particular, if $q_{0}\left[\rho^{\prime}\right\rangle v$, we have $w=h(v)$ and hence $\operatorname{Pre}(t) \leq h(v)$. As verified above, it follows that $\operatorname{Pre}(t)=h\left(v^{\prime}\right)$ for some multiset $v^{\prime} \leq v$. In particular, $v^{\prime}$ is covered in $S$ and by Proposition 4.5, there exists an event $e$ of $S$ such that $h(e)=t$ and $\ln (e)=v^{\prime}$. Therefore, the event $e$ is enabled by the marking $v$ and hence the sequence $\rho=\rho^{\prime} e$ is firable, which concludes the proof.

Note that, conversely, every firable sequence of an unfolding, or indeed of a branching process of a net $N$, maps to a firable sequence of $N$ (see Proposition 2.5).

Corollary 4.7. Every homomorphism between unfoldings of a net is surjective.

Proof. Let $g:(S, h) \rightarrow\left(S^{\prime}, h^{\prime}\right)$ be a homomorphism between two unfoldings of a net $N$. Since $S^{\prime}$ is quasi-live, every event occurs in a firing sequence enabled by its initial marking. So in order to establish that $g$ is surjective on events, it suffices to show that for each such firing sequence $\sigma^{\prime}, S$ has a firing sequence $\sigma$, enabled by its initial marking, and such that $g(\sigma)=\sigma^{\prime}$. We proceed by induction on the length of $\sigma^{\prime}$. 
If $\sigma^{\prime}$ is the empty sequence, it suffices to take $\sigma$ equal to the empty sequence. Suppose now that $\sigma^{\prime}=\tau^{\prime} e^{\prime}$, and let $m^{\prime}$ be the marking reached after firing $\tau^{\prime}$. In particular, $v^{\prime}=\ln \left(e^{\prime}\right)$ is covered by $m^{\prime}$. By induction, there exists a firing sequence $\tau$ in $S$ such that $g(\tau)=\tau^{\prime}$ : the marking reached after firing $\tau$ is $m=g\left(m^{\prime}\right)$. Then there exists a marking $v$ of $S$ such that $g(v)=v^{\prime}$ and $v$ is covered by $m$.

Moreover Pre $\left(h^{\prime}\left(e^{\prime}\right)\right)=h^{\prime}\left(v^{\prime}\right)=h(v)$ and Proposition 4.5 shows that there exists an event $e$ in $S$ such that $h(e)=h^{\prime}\left(e^{\prime}\right)$ and $\ln (e)=v$. Then $\ln (g(e))=g(v)=\ln \left(e^{\prime}\right)$ and $h^{\prime}\left(e^{\prime}\right)=h^{\prime}(g(e))$ : by definition of a branching process, $g(e)=e^{\prime}$.

Thus $g$ is surjective on events, and therefore also on the set of non-isolated conditions. In occurrence nets, the only isolated places are in the support of the initial marking, and every homomorphism is surjective on that set. This concludes the proof.

Corollary 4.8. If $(S, h)$ is an unfolding of a net $N$ and $\left(S^{\prime}, g\right)$ is an unfolding of $S$, then $\left(S^{\prime}, h \circ g\right)$ is an unfolding of $N$.

Proof. By Proposition 4.5, it suffices to show that if $t$ is a transition of $N$ and $\operatorname{Pre}(t)=h \circ g\left(v^{\prime}\right)$ for a marking $v^{\prime}$ that is covered in $S^{\prime}$, then there exists an event $e^{\prime}$ of $S^{\prime}$ such that $h \circ g\left(e^{\prime}\right)=t$ and $\ln \left(e^{\prime}\right)=v^{\prime}$. Then $g\left(v^{\prime}\right)$ is covered in $S$, so there exists an event $e$ of $S$ such that $h(e)=t$ and $\ln (e)=g\left(v^{\prime}\right)$ (by Proposition 4.5, since $(S, h)$ is an unfolding). And since $\left(S^{\prime}, g\right)$ is an unfolding of $S$, there exists an event $e^{\prime}$ of $S^{\prime}$ such that $g\left(e^{\prime}\right)=e$ and $\ln \left(e^{\prime}\right)=v^{\prime}$ (Proposition 4.5 again). Then $h \circ g\left(e^{\prime}\right)=t$, which concludes the proof.

\subsection{Quasi-order on branching processes and isomorphism}

In this section, we investigate the equivalence relation induced by the quasi-order $\preceq$. We show that $\preceq$ equivalent unfoldings of a net $N$ are isomorphic. We need an additional (but reasonable) hypothesis to establish the same result for all the branching processes of $N$.

Proposition 4.9. If $(S, h)$ and $\left(S^{\prime}, h^{\prime}\right)$ are unfoldings of a net $N$ and if $(S, h) \preceq\left(S^{\prime}, h^{\prime}\right)$, then $(S, h)$ and $\left(S^{\prime}, h^{\prime}\right)$ are isomorphic.

Proof. By definition, there exists an injective homomorphism $g:(S, h) \rightarrow\left(S^{\prime}, h^{\prime}\right)$. By Corollary 4.7, $g$ is surjective. Therefore $g$ is an isomorphism.

We say that a net has finite preconditions if the multisets $\operatorname{Pre}(t)$ (for every transition $t$ ) have finite support. We show in Corollary 4.13 that, under this assumption, $\preceq$-equivalent branching processes are isomorphic. We first record several technical results, the first of which is immediate from the definition.

Lemma 4.10. Let $h: N \rightarrow N^{\prime}$ be a homomorphism of nets. If $N^{\prime}$ has finite preconditions, then so does $N$. The converse holds if $h$ is onto.

Proposition 4.11. Let $(S, h)$ and $\left(S^{\prime}, h^{\prime}\right)$ be branching processes of a net $N$ with finite preconditions. If $S$ contains finitely many events and $(S, h) \preceq\left(S^{\prime}, h^{\prime}\right)$, then $\left(S^{\prime}, h^{\prime}\right)$ has only a finite number of prefixes that are isomorphic to $(S, h)$. 
Proof. We may assume without loss of generality that $(S, h)$ is actually a prefix of $\left(S^{\prime}, h^{\prime}\right)$. We proceed by induction on the cardinality of the set of events in $S$. If $S$ contains no events, then $S$ must be the unique eventless prefix of $S^{\prime}$, namely the support of the initial marking.

Now suppose that $S$ has at least one event, let $e$ be a maximal event in $S$ and let $S_{e}$ be the prefix of $S$ obtained by removing $e$ and $e^{\bullet}$ from $S$. By induction, $S^{\prime}$ has a finite collection of prefixes that are isomorphic to $S_{e}$. Let $\left(S^{\prime \prime}, h^{\prime \prime}\right)$ be such a prefix: it suffices to show that $\left(S^{\prime \prime}, h^{\prime \prime}\right)$ can be extended in only finitely many ways to a prefix isomorphic to $(S, h)$. Such an extension is determined by the addition of an event $e^{\prime}$ of $S^{\prime}$ such that the support of $\operatorname{In}^{\prime}\left(e^{\prime}\right)$ is contained in $S^{\prime \prime}$ and $h^{\prime}\left(e^{\prime}\right)=h(e)$. For each condition $b$ in the support of $\ln (e)$, we have seen (Lemma $2.4(2))$ that $h^{-1}(h(b))$ is finite. Since $\ln (e)$ has finite support, there are only finitely many sets of places in $S^{\prime \prime}$ which can occur in the support of $\operatorname{In}^{\prime}\left(e^{\prime}\right)$, and we conclude using the guarded injectivity in the definition of a branching process.

Example 4.12. A counter-example to the statement of Proposition 4.11 when the net does not have finite preconditions is as follows. Let $N$ be the net with places $p_{1}, p_{2}, \ldots$, each initially marked with 2 tokens, and a single transition $t$ with $\operatorname{Pre}(t)=\sum_{i>1} 1 \cdot p_{i}$. Let $\left(S^{\prime}, h^{\prime}\right)$ be the branching process of $N$ with conditions $\{b(i, 1), b(i, 2) \mid i \geq 1\}$, all of them initially marked 1 , with $h^{\prime}(b(i, 1))=h^{\prime}(b(i, 2))=p_{i}$; the events of $S^{\prime}$ are the (uncountably many) $e_{X}$, where $X=(X(i))_{i \geq 1}$ is a sequence with values in $\{1,2\}$, and $\operatorname{In}^{\prime}\left(e_{X}\right)=\sum_{i>1} 1 \cdot b(i, X(i))$. If $Y$ is the sequence constantly equal to 1 and $S$ contains the conditions of $S^{\prime}$ and the single event $e_{Y}$, then $(S, h) \sqsubseteq\left(S^{\prime}, h^{\prime}\right)$ and a branching process of $N$, which is isomorphic to uncountably many prefixes of $\left(S^{\prime}, h^{\prime}\right)$, namely all the prefixes containing all the conditions and a single event.

The following corollary extends the result of Proposition 4.9.

Corollary 4.13. Let $(S, h)$ and $\left(S^{\prime}, h^{\prime}\right)$ be branching processes of a net $N$ with finite preconditions. If $(S, h) \preceq\left(S^{\prime}, h^{\prime}\right)$ and $\left(S^{\prime}, h^{\prime}\right) \preceq(S, h)$, then $(S, h)$ and $\left(S^{\prime}, h^{\prime}\right)$ are isomorphic.

Proof. Again, we may assume that $(S, h)$ is a prefix of $\left(S^{\prime}, h^{\prime}\right)$. In addition, there exists an injective homomorphism $g:\left(S^{\prime}, h^{\prime}\right) \rightarrow(S, h)$. We want to show that $g$ is an isomorphism, that is, $g$ is onto. Let $e$ be an event in $S$ and let $S_{e}$ be the least prefix of $S$ containing $e$. Note that $S_{e}$ contains finitely many events (namely the transitions in the past of $e$ ). By the first statement, there are finitely many prefixes of $S^{\prime}$ that are isomorphic to $S_{e}$, and therefore the injective homomorphism $g$ induces a permutation of these prefixes. In particular, $S_{e}=g\left(S_{e}^{\prime}\right)$ for one such prefix $S_{e}^{\prime}$ and hence $e \in g\left(S^{\prime}\right)$.

\section{Faithful unfolding and weakly safe unfolding of a net}

The main results of this section, Theorems 5.2 and 5.6, show the existence and the unicity (up to isomorphism) of faithful and weakly safe unfoldings.

\subsection{The faithful case}

We first record a crucial property of faithful unfoldings.

Proposition 5.1. Let $(S, h)$ be a branching process of a net $N$ and let $\left(S^{\prime}, h^{\prime}\right)$ be a faithful unfolding of $N$. Then there exists a unique homomorphism $g:(S, h) \rightarrow\left(S^{\prime}, h^{\prime}\right)$. 
Proof. We first establish the result when $S$ has finitely many events, proceeding by induction on the number of events in $S$. If $S$ has no event, $S$ coincides with its set of initial conditions, which is mapped by $h$ to the support of $m_{0}$. And by definition of faithful branching processes, $h^{\prime}$ establishes a bijection between the supports of $q_{0}^{\prime}$ and $m_{0}$ : the announced homomorphism is thus uniquely determined.

Let us now assume that $S$ contains some events, and let $e$ be a maximal event of $S$. Let $S_{e}$ be the prefix of $S$ obtained by removing $e$ and $e^{\bullet}$. In particular the support of $\ln (e)$ is contained in $S_{e}$, and the marking $\ln (e)$ is covered by a reachable marking of $S_{e}$ (since $S$ is quasi-live). By induction, there exists a homomorphism $g:\left(S_{e}, h\right) \rightarrow\left(S^{\prime}, h^{\prime}\right)$. Moreover, by Proposition 4.5, there exists an event $e^{\prime}$ of $S^{\prime}$ such that $h^{\prime}\left(e^{\prime}\right)=h(e)$ and $\ln \left(e^{\prime}\right)=g(\ln (e))$. We can now extend the homomorphism $g$ to $S$ by letting $g(e)=e^{\prime}$ and, for each condition $b \in e^{\bullet}, g(b)=h^{\prime-1}(h(b)) \cap e^{\prime \bullet}$ (which is well defined since the faithful unfolding $\left(S^{\prime}, h^{\prime}\right)$ establishes a bijection between $e^{\prime \bullet}$ and $\left.h(e)^{\bullet}\right)$.

Now let $(S, h)$ be an arbitrary branching process. For each element $x$ of $S$, event or condition, let $S_{x}$ be the least prefix of $S$ containing $x$. Then $S_{x}$ contains finitely many events (Proposition 3.1) and hence, there exists a homomorphism $g_{x}:\left(S_{x}, h\right) \rightarrow\left(S^{\prime}, h^{\prime}\right)$, and we let $g(x)=g_{x}(x)$. In order to show that $g$ is a homomorphism, it suffices to show that the restriction of $g$ to $S_{x}$ is exactly $g_{x}$, for every $x$. Let then $y$ be an element of $S_{x}$. We have $S_{y} \sqsubseteq S_{x}$ and two homomorphisms are defined from $\left(S_{y}, h\right)$ to $\left(S^{\prime}, h^{\prime}\right)$, namely $g_{y}$ and the restriction of $g_{x}$ to $S_{y}$. Lemma 4.4 (3) guarantees that these two homomorphisms coincide, which concludes the verification that $g$ is a homomorphism.

Lemma 4.4 (3) again establishes the uniqueness of $g$.

We can now state and prove the following theorem.

Theorem 5.2. Every net $N$ has a faithful unfolding $\mathcal{B}_{\text {faithful }}$, which is unique up to isomorphism. In addition, every branching process $(S, h)$ of $N$ admits a homomorphism to $\mathcal{B}_{\text {faithful }}$, and every faithful branching process satisfies $(S, h) \preceq \mathcal{B}_{\text {faithful }}$.

Proof. Let $N=\left(P, T\right.$, Pre, Post, $\left.m_{0}\right)$. We first consider the net $S_{0}$, whose condition set is $\overline{m_{0}}$, with initial marking $m_{0}$, and without any event. Then $S_{0}$ is an occurrence net and if $h_{0}$ is the identity map on $\overline{m_{0}}$, then $\left(S_{0}, h_{0}\right)$ is a faithful branching process of $N$.

Suppose that we have constructed a sequence $\left(S_{i}, h_{i}\right)_{i \leq n}$ of faithful branching processes, such that $\left(S_{i}, h_{i}\right) \sqsubseteq\left(S_{j}, h_{j}\right)$ whenever $i<j$. By definition, $S_{i}$ is a subnet (and a prefix) of $S_{j}$ and $h_{i}$ is the restriction to $S_{i}$ of $h_{j}$. A new net $S_{n+1}$ is constructed from $S_{n}$ as follows.

For each transition $t$ of $N$ and for each marking $v$ of $S_{n}$, covered in $S_{n}$, such that $\operatorname{Pre}(t)=h_{n}(v)$, and such that $S_{n}$ has no event $e$ with $h_{n}(e)=t$ and $\ln _{n}(e)=v$, we add to $S_{n}$ a new event $e$, and a set of new conditions $Q$, equipped with a bijection $\theta$ onto the support of $\operatorname{Post}(t)$ ( $e$ and the set $Q$ depend on the choice of $t$ and $v$ ). Then we extend the mappings $\ln _{n}$ and Out ${ }_{n}$ by letting $\ln _{n+1}(e)=v$ and Out $_{n+1}(e)=\sum_{q \in Q} \operatorname{Post}(\theta(q), t) q$ for each new event. We also extend $h_{n}$ by letting $h_{n+1}(e)=t$ for each new event $e$, and $h_{n+1}(q)=\theta(q)$ for each new condition $q$. It is immediate that the resulting net $S_{n+1}$ is an occurrence net, that the pair $\left(S_{n+1}, h_{n+1}\right)$ is a faithful branching process of $N$, and that $\left(S_{n}, h_{n}\right) \sqsubseteq\left(S_{n+1}, h_{n+1}\right)$.

Note that $S_{n+1}$ may not be defined (if no pair $(t, v)$ as above can be identified in $S_{n}$ ), in which case the sequence $\left(S_{i}, h_{i}\right)_{i}$ is finite. In general however, this is an infinite increasing sequence of faithful branching processes of $N$. In any case, let $\mathcal{B}_{\text {faithful }}=\left(B, E, \ln\right.$, Out, $\left.q_{0}\right)$ be the union of the $S_{i}$, that is, $B=\bigcup_{i \in I} B_{i}$ and $E=\bigcup_{i \in I} E_{i}$, with $\operatorname{In}(e)=\operatorname{In}_{i}(e)$ and $\operatorname{Out}(e)=\operatorname{Out}_{i}(e)$ if $e \in E_{i}$. The mappings 
$h_{\text {faithful }}: B \rightarrow P$ and $h_{\text {faithful }}: E \rightarrow T$ are defined similarly, by letting $h_{\text {faithful }}(x)=h_{i}(x)$ whenever $x$ occurs in $S_{i}$. Again, it is easily verified that $\left(\mathcal{B}_{\text {faithful }}, h_{\text {faithful }}\right)$ is a faithful branching process of $N$. We now verify that it is an unfolding, using Condition $(\dagger)$ in Proposition 4.5.

Let $t$ be a transition of $N$ such that $\operatorname{Pre}(t)=h_{\text {faithful }}(v)$ for some marking $v$ covered in $\mathcal{B}_{\text {faithful }}$. Then $v$ is covered in some $S_{n}$ and $\operatorname{Pre}(t)=h_{n}(v)$. By construction, there exists an event $e$ in $S_{n}$ or in $S_{n+1}$ such that $h_{n+1}(e)=t$ and $\ln _{n+1}(e)=v$. In particular $h_{\text {faithful }}(e)=t$ and $\ln (e)=v$, so Condition $(\dagger)$ holds and $\left(\mathcal{B}_{\text {faithful }}, h_{\text {faithful }}\right)$ is an unfolding.

Let now $(S, h)$ be a branching process of $N$. By Proposition 5.1, there exists a homomorphism $g:(S, h) \rightarrow \mathcal{B}_{\text {faithful. If }}(S, h)$ is faithful, then $g$ is injective by Lemma 4.4 , that is, $(S, h) \preceq \mathcal{B}_{\text {faithful }}$. Finally, if $(S, h)$ is another faithful unfolding, we conclude by Proposition 4.9.

Theorem 5.2 and Lemma 4.4 (3) and (4) imply the following illuminating observation.

Corollary 5.3. Each faithful branching process of a net $N$ is isomorphic to a single prefix of the faithful unfolding of $N$.

Corollary 5.3 shows the following, more detailed order-theoretic result.

Proposition 5.4. The set of faithful branching processes of a net $N$ forms a complete lattice.

Proof. Let $\left(S_{i}, h_{i}\right)_{i \in I}$ be a family of faithful branching processes of $N$. By Corollary 5.3, each $S_{i}$ can be identified with a subnet (and hence a prefix, Lemma 3.2) of $\mathcal{B}_{\text {faithful }}$. The announced result now follows from the fact that prefixes of a net, and ultimately ideals of an ordered set, form a complete lattice.

\subsection{The weakly safe case}

We get a result analogous to Theorem 5.2 on the existence of a weakly safe unfolding, but the ordertheoretic properties of weakly safe branching processes are less strong than for faithful branching processes.

Proposition 5.5. Let $(S, h)$ be a weakly safe branching process of a net $N$ and let $\left(S^{\prime}, h^{\prime}\right)$ be a weakly safe unfolding of $N$. Then $(S, h) \preceq\left(S^{\prime}, h^{\prime}\right)$.

Proof. Let $S_{n}$ be the least prefix of $S$ containing the events with at most $n$ transitions in their past. Then $S_{n} \sqsubseteq S_{n+1}$ and $S$ is the union of the $S_{n}$ by Proposition 3.1.

$S_{0}$ coincides with $q_{0}$, the initial conditions of $S$, which is mapped by $h$ to $m_{0}$. Similarly, $h^{\prime}$ maps the initial conditions $q_{0}^{\prime}$ of $S^{\prime}$ to $m_{0}$ : thus, for every initial place $p$ of $N, h^{-1}(p)$ and $h^{\prime-1}(p)$ have the same number of elements, namely $m_{0}(p)$, and one can define accordingly an injective homomorphism $g:\left(S_{0}, h\right) \rightarrow\left(S^{\prime}, h^{\prime}\right)$.

Let us now assume that $g:\left(S_{n-1}, h\right) \rightarrow\left(S^{\prime}, h^{\prime}\right)$ is an injective homomorphism and let $e$ be an event of $S_{n}$ not in $S_{n-1}$. Then the support of $\ln (e)$ is contained in $S_{n-1}$ and the marking $v=\ln (e)$ is covered by a reachable marking of $S_{n-1}$ (since $S$ is quasi-live). Since Pre $(h(e))=h(v)=h^{\prime}(g(v))$, Proposition 4.5 shows that there exists an event $e^{\prime}$ of $S^{\prime}$ such that $h^{\prime}\left(e^{\prime}\right)=h(e)$ and $\ln \left(e^{\prime}\right)=g(v)$. This event is unique, by definition of a branching process and we let $g(e)=e^{\prime}$. 
Now $h$ (resp. $h^{\prime}$ ) maps $e^{\bullet}$ (resp. $e^{\boldsymbol{\bullet}^{\bullet}}$ ) to $h(e)^{\bullet}$. For each place $p$ in $h(e)^{\bullet}$, the sets $h^{-1}(p) \cap e^{\bullet}$ and $h^{\prime-1}(p) \cap e^{\prime \bullet}$ have the same cardinality, namely Post $(p, h(e))$ (since $S$ and $S^{\prime}$ are weakly safe), and $g$ can be extended accordingly to an injective homomorphism from $\left(S_{n}, h\right)$ to $\left(S^{\prime}, h^{\prime}\right)$.

Since $S$ is the union of the $S_{n}$ (Proposition 3.1), this establishes the existence of an injective homomorphism from $(S, h)$ to $\left(S^{\prime}, h^{\prime}\right)$, and hence $(S, h) \preceq\left(S^{\prime}, h^{\prime}\right)$.

Theorem 5.6. Every net $N$ has a weakly safe unfolding $\mathcal{B}_{\text {w.safe }}$ which is unique up to isomorphism. In addition, every weakly safe branching process $(S, h)$ satisfies $(S, h) \preceq \mathcal{B}_{\text {w.safe }}$.

Proof. The proof of the existence of a weakly safe unfolding follows the same line as that of Theorem 5.2, and we only indicate the differences. As in the proof of Theorem 5.2, the net $S_{0}$ has no event, and only initial conditions ; but its condition set is defined by splitting each initial place of $N$ according to its initial marking. More precisely, $S_{0}$ consists of a disjoint union $\bigcup_{p \in \overline{m_{0}}} B^{p}$ where each set $B^{p}$ consists of $m_{0}(p)$ elements, each with an initial marking equal to 1 . The map $h_{0}$ sends each condition in $B^{p}$ to $p$ : thus $\left(S_{0}, h_{0}\right)$ is a weakly safe branching process of $N$.

Again, we assume that we have constructed a finite sequence of weakly safe branching processes $\left(S_{i}, h_{i}\right)_{i \leq n}$, where $\left(S_{i}, h_{i}\right) \sqsubseteq\left(S_{j}, h_{j}\right)$ for each $i<j$. If $t$ and $v$ are a transition of $N$ and a marking of $S_{n}$ as in the proof of Theorem 5.2, we construct $\left(S_{n+1}, h_{n+1}\right)$ from $\left(S_{n}, h_{n}\right)$ as in that proof, with the following difference: the set $Q$ of new conditions added to $S_{n}$ in relation with the pair $(t, v)$ is the disjoint union $\bigcup_{p \in \overline{\operatorname{Post}(t)}} Q^{p}$ of sets such that $Q^{p}$ has Post $(t, p)$ elements. Then Out ${ }_{n+1}(e)=\sum_{p \in \overline{\operatorname{Post}(t)}} \sum_{q \in Q^{p}} q$, and $h_{n+1}$ maps each condition in $Q^{p}$ to the place $p$.

It is immediately verified that $\left(S_{n+1}, h_{n+1}\right)$ is a weakly safe branching process of $N$. Now, if $\left(\mathcal{B}_{\text {w.safe }}, h_{\text {w.safe }}\right)$ is defined as in the proof of Theorem 5.2, as the inductive limit of the increasing sequence of branching processes $\left(S_{n}, h_{n}\right)$, then $\left(\mathcal{B}_{\text {w.safe }}, h_{\text {w.safe }}\right)$ is a weakly safe unfolding of $N$.

Now let $(S, h)$ be a weakly safe branching process of $N$. Proposition 5.5 shows that $(S, h) \preceq$ $\mathcal{B}_{\text {w.safe. }}$ If in addition $(S, h)$ is an unfolding, we conclude that $(S, h)$ and $\mathcal{B}_{\text {w.safe }}$ are isomorphic by Proposition 4.9.

However, a weakly safe branching process of $N$ cannot be identified unequivocally with a prefix of $\mathcal{B}_{\text {w.safe }}$, and we don't have an analogue of Proposition 5.4 for weakly safe branching processes. This is illustrated in the following example.

Example 5.7. Consider the net $N$ represented in Figure 4. The nets $\beta_{1}, \ldots, \beta_{4}$ represented in Figure 5 are weakly safe branching processes of $N$. Moreover, it is easy to verify that both $\beta_{3}$ and $\beta_{4}$ are $\sqsubseteq$-less than $\beta_{1}$ and $\beta_{2}$, and that both are maximal with this property: thus $\beta_{1}$ and $\beta_{2}$ do not admit a greatest lower bound. Similarly, Figure 6 shows weakly safe branching processes $\beta_{1}$ and $\beta_{2}$, and distinct branching processes $\beta_{3}$ and $\beta_{4}$ which are minimal among the common upper bounds of $\beta_{1}$ and $\beta_{2}$. Thus $\beta_{1}$ and $\beta_{2}$ do not admit a least upper bound. Note that these weakly safe branching processes are also safe.

It is interesting to observe (compare with Corollary 5.3) that in both cases, both $\beta_{1}$ and $\beta_{2}$ are isomorphic to two distinct subnets of the weakly safe unfolding of $N$, represented in Figure 7.

\subsection{Comparing unfoldings}

Let us first reiterate that safe or weakly safe nets have a single unfolding. Indeed, we saw in Proposition 4.1 that all the branching processes of a safe (resp. weakly safe) net have the same property. Recall 


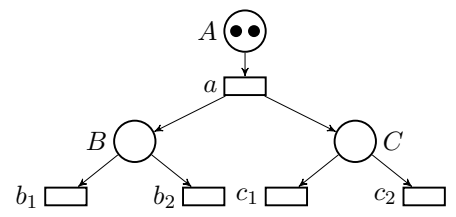

Figure 4. The net $N$ in Example 5.7

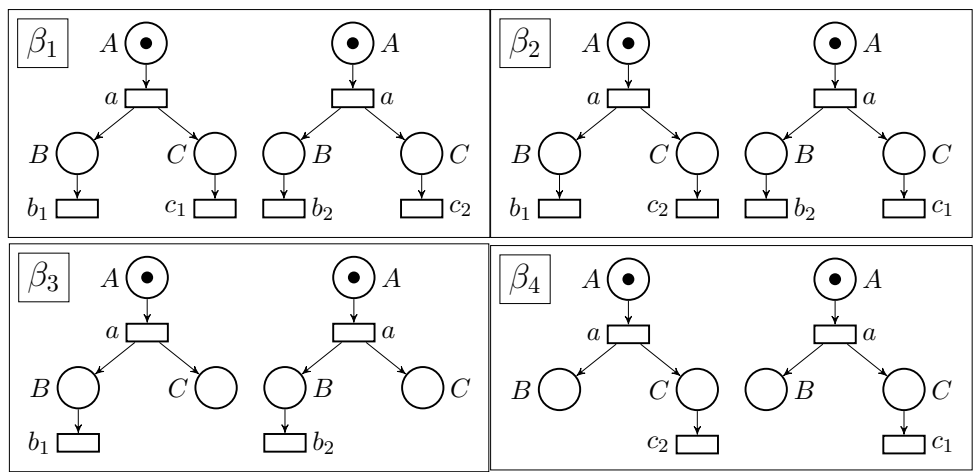

Figure 5. Weakly safe branching processes may not have an inf

also that, in general, a weakly safe branching process (or unfolding) is safe if it contains no spontaneous, non-isolated transition (Proposition 3.3).

We now consider general nets, which may have several unfoldings. Let us write $(S, h) \Longrightarrow\left(S^{\prime}, h^{\prime}\right)$ if $(S, h)$ and $\left(S^{\prime}, h^{\prime}\right)$ are unfoldings of a net $N$ and there exists a homomorphism $g:\left(S^{\prime}, h^{\prime}\right) \rightarrow(S, h)$. (This is equivalent to $(S, g)$ being a branching process of $S^{\prime}$ by Lemma 4.4 (1).) Note that $g$ is not required to be injective, but it is surjective by Corollary 4.7. The relation $\Longrightarrow$ is a quasi-order on the set of unfoldings of $N$ and we shall verify that the faithful unfolding and the weakly safe unfolding of a net $N$ sit at extreme positions with respect to this order.

Remark 5.8. An informal way of viewing the $\Longrightarrow$ relation between unfoldings is the following: the existence of a homomorphism from $(S, h)$ to $\left(S^{\prime}, h^{\prime}\right)$ can be understood as saying that $\left(S^{\prime}, h^{\prime}\right)$ is more "folded" than $(S, h)$. From this point of view, the results of this Section state that the weakly safe unfolding of $N$ is its least folded unfolding, whereas its faithful unfolding is its most folded unfolding.

First we show that $\Longrightarrow$-equivalent unfoldings are isomorphic.

Lemma 5.9. Let $(S, h)$ be a branching process of a net $N$ and let $g:(S, h) \rightarrow(S, h)$ be a homomorphism. Then $g$ is injective on every prefix of $S$ with a finite number of events.

Proof. Let $S^{\prime}$ be a prefix of $S$ with finitely many events, and let $b$ be a condition of $S^{\prime}$. Then $g$ induces a surjective mapping from the set of elements of $h^{-1}(h(b))$ in $S^{\prime}$, to the set of elements of $h^{-1}(h(b))$ in $g\left(S^{\prime}\right)$. Since $S$ is an occurrence net (and therefore has no non-initally marked, isolated place), the set $h^{-1}(h(b))$ has finitely many elements in $S^{\prime}$ by Lemma 2.4 (2). So $g$ actually induces a bijection on the 

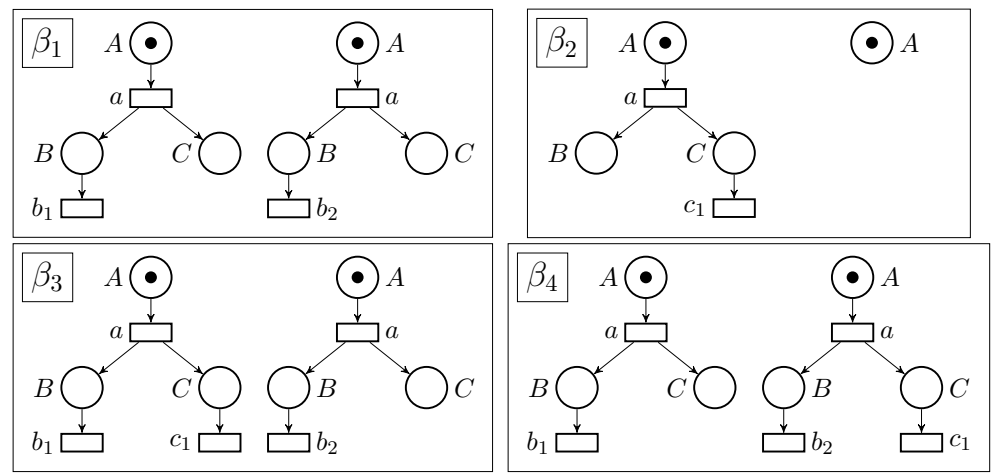

Figure 6. Weakly safe branching processes may not have a sup

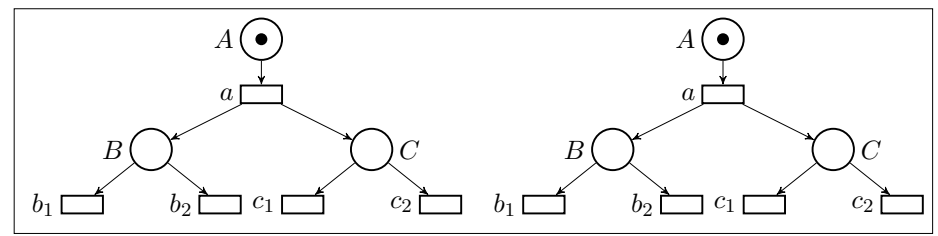

Figure 7. The weakly safe unfolding of the net $N$ in Example 5.7

subset of $h^{-1}(h(b))$ in $S^{\prime}$. This implies that the restriction of $g$ to $S^{\prime}$ is injective on conditions: indeed, if $g(b)=g(c)$, then $h(b)=h(g(b))=h(g(c))=h(c)$, that is, $c \in h^{-1}(h(b))$.

Let now $e$ and $f$ be events in $S^{\prime}$ such that $g(e)=g(f)$. Then $h(e)=h(f)$ and $g(\ln (e))=g(\ln (f))$. The last equality implies that $\ln (e)=\ln (f)$, and the guarded injectivity in the definition of branching processes then guarantees that $e=f$. This concludes the proof.

Proposition 5.10. Let $(S, h)$ and $\left(S^{\prime}, h^{\prime}\right)$ be unfoldings of a net $N$ such that $(S, h) \Longrightarrow\left(S^{\prime}, h^{\prime}\right) \Longrightarrow$ $(S, h)$. Then $(S, h)$ and $\left(S^{\prime}, h^{\prime}\right)$ are isomorphic.

Proof. Let $g:(S, h) \rightarrow\left(S^{\prime}, h^{\prime}\right)$ and $g^{\prime}:\left(S^{\prime}, h\right) \rightarrow(S, h)$ be homomorphisms. Then $g$ is surjective by Corollary 4.7. Let now $x, y$ be elements of $S$, events or conditions, such that $g(x)=g(y)$. Let $S^{\prime \prime}$ be a prefix of $S$ containing $x$ and $y$, with finitely many events: such a prefix exists by Proposition 3.1. Then $g^{\prime} \circ g$ is injective on $S^{\prime \prime}$ by Lemma 5.9, and hence $g$ is injective on $S^{\prime \prime}$ : it follows that $x=y$. $S$ to $g(S)=S^{\prime}$.

Theorem 5.11. The faithful unfolding of a net is its unique $\Longrightarrow$-minimal unfolding (up to isomorphism).

Proof. By Theorem 5.2, every unfolding $(S, h)$ of $N$ admits a homomorphism $g^{\prime}:(S, h) \rightarrow \mathcal{B}_{\text {faithful }}$, that is, $\mathcal{B}_{\text {faithful }} \Longrightarrow(S, h)$. If $(S, h) \Longrightarrow \mathcal{B}_{\text {faithful }}$, that is, if there exists a homomorphism $g^{\prime}: \mathcal{B}_{\text {faithful }} \rightarrow$ $(S, h)$, then $g \circ g^{\prime}$ is a homomorphism from $\mathcal{B}_{\text {faithful }}$ to $\mathcal{B}_{\text {faithful }}$ and Lemma 4.4 (3) shows that $g \circ g^{\prime}$ is the identity of $\mathcal{B}_{\text {faithful }}$ and hence, $\mathcal{B}_{\text {faithful }}$ and $(S, h)$ are isomorphic.

Theorem 5.12. The weakly safe unfolding of a net is its unique $\Longrightarrow$-maximal unfolding (up to isomorphism). 
Proof. If $(S, h)$ is an unfolding of $N$ and $\left(S^{\prime}, g\right)$ is a weakly safe unfolding of $S$, then $\left(S^{\prime}, h \circ g\right)$ is a weakly safe unfolding of $N$ by Corollary 4.8 and hence, $\left(S^{\prime}, h \circ g\right)$ is isomorphic to $\mathcal{B}_{\text {w.safe }}$ (Theorem 5.6). Moreover, $g:\left(S^{\prime}, h \circ g\right) \rightarrow(S, h)$ is a homomorphism, so we have $(S, h) \Longrightarrow \mathcal{B}_{\text {w.safe }}$.

Now if $\mathcal{B}_{\text {w.safe }} \Longrightarrow(S, h)$, there exists a surjective homomorphism from $(S, h)$ to $\mathcal{B}_{\text {w.safe }}$, and this implies that $(S, h)$ is weakly safe. Therefore $(S, h)$ is isomorphic to $\mathcal{B}_{\text {w.safe. }}$

\section{Processes and their properties}

The notion of process of a net is developped in the literature [13,3], but it is limited to the case of safe processes. Here we extend the notion, in such a way that the usual processes are what we call here safe processes.

By definition, a process of a net $N$ is a triple $\pi=(S, h, \varphi)$ such that $(S, h)$ is a branching process of $N$, and $\varphi$ is a configuration of $S$ which covers all its events (that is, every event of $S$ is in the support of $\varphi)$. We say that a process $\pi=(S, h, \varphi)$ is faithful (resp. safe, weakly safe) if the underlying branching process $(S, h)$ is faithful (resp. safe, weakly safe). Moreover, if $\left(S^{\prime}, h^{\prime}\right)$ is a branching process of $N$ such that $(S, h) \preceq\left(S^{\prime}, h^{\prime}\right)$, we say that $\pi$ is a process of the branching process $\left(S^{\prime}, h^{\prime}\right)$.

Finally, we extend the quasi-order on branching processes of $N$, to its processes. If $\pi_{1}=\left(S_{1}, h_{1}, \varphi_{1}\right)$ and $\pi_{2}=\left(S_{2}, h_{2}, \varphi_{2}\right)$ are processes of a net $N$, we say that $\pi_{1}$ is smaller than $\pi_{2}$, written $\pi_{1} \preceq \pi_{2}$, if there exists an injective homomorphism $g:\left(S_{1}, h_{1}\right) \rightarrow\left(S_{2}, h_{2}\right)$ with $g\left(\boldsymbol{\varphi}_{1}\right) \leq \boldsymbol{\varphi}_{2}$.

Remark 6.1. Our processes differ from those defined by Engelfriet in the weakly safe case: by definition, our processes have finitely many events, whereas Engelfriet's definition allows an infinite number of events.

\subsection{Faithful processes}

The above definitions, together with Theorems 5.2 and 5.6, yield immediately the following result.

Proposition 6.2. Every faithful (resp. weakly safe) process of a net $N$ is a process of the faithful (resp. weakly safe) unfolding of $N$.

Proposition 6.2 then leads to the following characterization of the faithful unfolding of a net.

Proposition 6.3. Let $(S, h)$ be a branching process of a net $N$. If every faithful process of $N$ is a process of $(S, h)$, then $(S, h)$ is isomorphic to the faithful unfolding of $N$.

Proof. We first assume that $(S, h)$ is faithful. If $\left(S^{\prime}, h^{\prime}, \varphi\right)$ is a faithful process of $N$, then $\left(S^{\prime}, h^{\prime}\right)$ is isomorphic to a uniquely determined prefix of $(S, h)$ by Proposition 5.1.

We apply this fact to the prefixes of $\mathcal{B}_{\text {faithful }}$ : let $e$ be an event of $\mathcal{B}_{\text {faithful }}$. Since $\mathcal{B}_{\text {faithful }}$ is quasilive, $e$ occurs in a configuration $\varphi$ of $\mathcal{B}_{\text {faithful }}$. Let $S_{e}$ be the least prefix of $\mathcal{B}_{\text {faithful }}$ containing the events in the support of $\boldsymbol{\varphi}$, and let $h_{e}$ be the restriction of $h_{\text {faithful }}$ to $S_{e}$. Then the process $\left(S_{e}, h_{e}, \varphi\right)$ is a faithful process of $N$. Therefore $\left(S_{e}, h_{e}\right)$ is isomorphic to a single prefix of $(S, h)$, and we let $g_{e}:\left(S_{e}, h_{e}\right) \rightarrow(S, h)$ be the corresponding injective homomorphism. In particular, if $e$ and $f$ are events of $\mathcal{B}_{\text {faithful }}$, then $g_{e}$ and $g_{f}$ coincide on the intersection of $S_{e}$ and $S_{f}$. Therefore the injective homomorphisms $g_{e}$ (when $e$ runs over all the events of $\mathcal{B}_{\text {faithful }}$ ) induce an injective homomorphism 
$g: \mathcal{B}_{\text {faithful }} \rightarrow(S, h)$, that is, $\mathcal{B}_{\text {faithful }} \preceq(S, h)$. It follows from Theorem 5.2 that $(S, h)$ is isomorphic to $\mathcal{B}_{\text {faithful }}$.

In order to conclude, we now show that $(S, h)$ is necessarily faithful. Indeed, if this is not the case, we consider the subnet $R$ of $S$ which consists of the initial conditions, of the events $e$ such that, for each event $f \leq e, h$ is injective on $f^{\bullet}$, and of the pre- and post-conditions of these events. It is immediate that $R$ is a prefix of $S$ and therefore an occurrence net (Lemma 3.2). If $k$ is the restriction of $h$ to $R$ then $(R, k) \sqsubseteq(S, h)$. Moreover, $(R, k)$ is a faithful branching process by construction, and every faithful process of $(S, h)$ is in fact a faithful process of $(R, k)$. It follows from the first part of the proof that $(R, k)$ is isomorphic to $\mathcal{B}_{\text {faithful }}$, and since $\mathcal{B}_{\text {faithful }}$ is $\preceq$-maximal, $(S, h)$ is isomorphic to $\mathcal{B}_{\text {faithful }}$ as well (Proposition 4.9), a contradiction.

We can also show that faithful processes have valuable order-theoretic properties with respect to the $\preceq$-order.

Proposition 6.4. Any family of faithful processes of a net $N$ admits a greatest lower bound, which is a faithful process as well. If a family of faithful processes of $N$ admits a common upper bound which is faithful, then it has a least upper bound among faithful processes.

Proof. Let $\left(\pi_{i}\right)_{i \in I}$ be a family of faithful processes of $N$, with $\pi_{i}=\left(S_{i}, h_{i}, \varphi_{i}\right)$ for each $i$. Each $\left(S_{i}, h_{i}\right)$ can be viewed in an unambiguous fashion as a prefix of $\mathcal{B}_{\text {faithful }}$ (Corollary 5.3), and hence $\varphi_{i}$ can be viewed as a configuration of $\mathcal{B}_{\text {faithful }}$. Let $\varphi=\min _{i} \varphi_{i}$, that is, $\varphi=\sum_{e} \min _{i} \varphi_{i}(e) \cdot e$, where the sum runs over all the events of $\mathcal{B}_{\text {faithful }}$. Let $S$ be the subnet of $\mathcal{B}_{\text {faithful }}$ consisting of the initial conditions, the events occurring in $\varphi$ and their pre- and post-conditions. It is easily verified that $S$ is a prefix of $\mathcal{B}_{\text {faithful }}$, and if $h$ is the restriction of (any) $h_{i}$ to $S,(S, h)$ is a faithful branching process of $N$. It is also clear that $(S, h) \sqsubseteq\left(S_{i}, h_{i}\right)$ and $\boldsymbol{\varphi} \leq \boldsymbol{\varphi}_{i}$ for each $i$. We want to show that $(S, h, \varphi)=\min _{i} \pi_{i}$, and for this purpose, it suffices to establish that $\varphi$ is a configuration. We use the characterization given in Corollary 3.5.

First it is immediate that $\varphi$ has finite support, since each $\varphi_{i}$ does. Moreover, the fact that each $\varphi_{i}$ satisfies the inequalities in Corollary 3.5 easily implies that $\varphi$ does as well. Thus $\varphi$ is a configuration and $(S, h, \varphi)=\min _{i} \pi_{i}$.

The statement concerning upper bounds follows immediately: if the set $U$ of faithful upper bounds of $\left(\pi_{i}\right)_{i \in I}$ is non-empty, we claim that $\inf U$ is the least upper bound of the $\left(\pi_{i}\right)_{i \in I}$. To justify this claim, it suffices to verify that inf $U$ is indeed an upper bound, that is, inf $U \in U$. This is readily verified, using the description of inf $U$ in the first part of the proof, and the fact that the configurations we consider are finite support vectors with positive integer coefficients.

Remark 6.5. Let $\left(\pi_{i}\right)_{i \in I}$ be a family of faithful processes of a net $N$ as in Proposition 6.4. It is not difficult to verify that this family does not have an upper bound if it takes infinitely many values. Now consider the net $N$ in Figure 4, and consider the faithful processes determined by the configurations $a+b_{1}, a+b_{2}$ and $a+c_{1}$. Any two of these processes have a least upper bound: for instance $\sup (a+$ $\left.b_{1}, a+b_{2}\right)=2 a+b_{1}+b_{2}$. Note in particular that the sup of these configurations taken as multisets, $a+b_{1}+b_{2}$, is not a configuration. Moreover, the three processes taken together do not admit a common upper bound. 


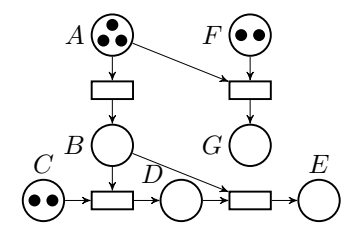

Figure 8. The net $N$ in Section 6.2

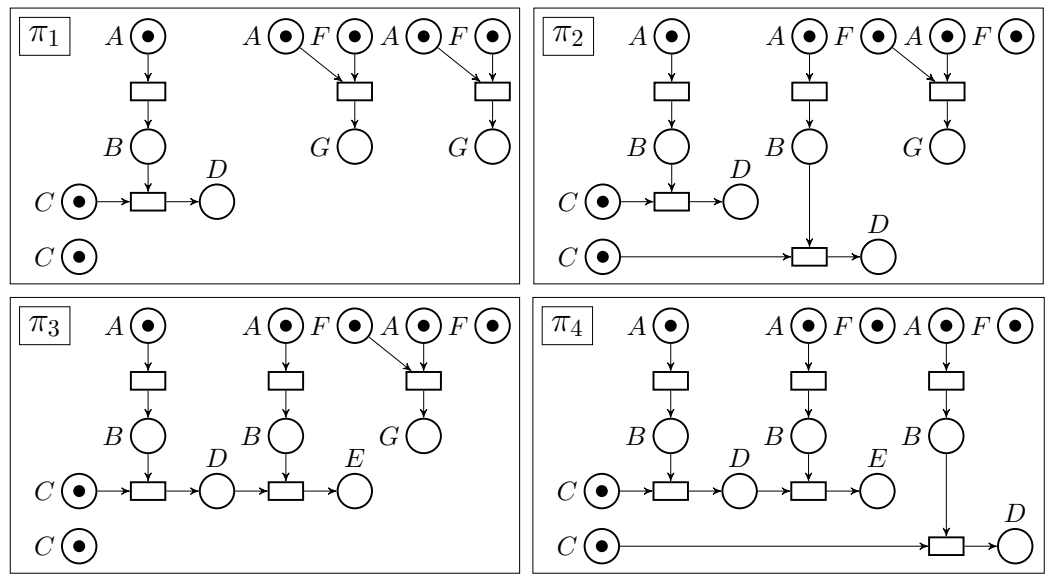

Figure 9. All the maximal weakly safe processes of the net $N$ in Section 6.2

\subsection{Weakly safe processes}

In this section, we verify that weakly safe processes do not have the good order-theoretic properties enjoyed by faithful processes, described in Propositions 6.3 and 6.4.

Consider the net $N$ represented in Figure 8 . Figure 9 shows all the $\preceq$-maximal weakly safe processes of $N$ (up to isomorphism): there are 4 of them.

Moreover, Figure 10 shows two distinct weakly safe branching processes of $N$, both of which contain all the maximal weakly safe processes. Thus the analogue of Proposition 6.3 does not hold for weakly safe processes. Example 5.7 demonstrates that Proposition 6.4 does not hold for weakly safe processes.

Of course, the weakly safe branching processes of Figure 10 also occur in the weakly safe unfolding of $N$, shown in Figure 11. This weakly safe unfolding exhibits large-scale duplication of weakly safe processes.

\section{Concluding remarks}

This paper proposes a general framework for the unfolding of general Petri nets. Traditional occurrence nets are covered by safe unfoldings. When applied to general nets, this approach has certain drawbacks, which are exemplified in Sections 5.2 and 6.2: even though every net admits a unique weakly safe unfolding (which is safe unless the net contains spontaneous non-isolated transitions), the safe branching processes do not form a lattice, and neither do the safe processes. The root of the problem can be traced to the following fact: a safe process of a net $N$ may occur in several ways as a process of the weakly safe 


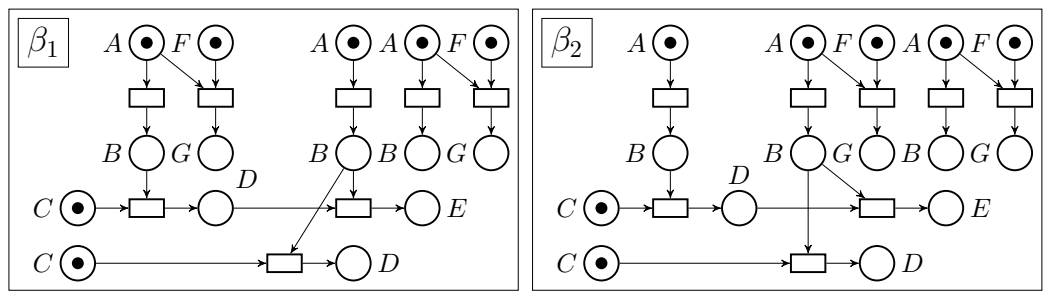

Figure 10. Two weakly safe branching processes containing all the weakly safe processes of the net $N$ in Section 6.2

unfolding of $N$.

The essential contribution of our work is the concept of faithful unfolding of a net. This is an extension of the traditional notion in the following sense: if $N$ is a safe Petri net, then its weakly safe and its faithful unfoldings (resp. branching processes, processes) coincide. The faithful processes and the faithful branching processes of a general net satisfy good order-theoretic properties. Moreover, faithful branching processes as well as faithful processes have a unique representation in the faithful unfolding.

Another contribution of the paper is the absence of finiteness conditions on our nets: neither on the number of places or transitions, nor on the support of the pre- and post-conditions of the transitions. To go further, it would be interesting to investigate the maximal behaviors of infinite nets. In particular, a transition with an infinite number of non-initially marked pre-conditions could become firable after firing an infinite number of transitions within a finite amount of time.

One question not tackled in this paper is the relationship between unfoldings and event structures. Within the framework of general Petri nets, the concept of prime event structure is not adapted to capture the multiset aspects. Two natural questions should be considered: (1) In which cases does the notion of faithful unfolding make it possible to capture the conflict and causality relations? (2) What is the concept of event structures associated to faithful unfoldings? An additional question concerns the interpretation of faithful unfoldings to define a true concurrency semantics similarly to van Glabbeek and Plotkin [4].

A natural follow-up of our work would be to use finite faithful branching processes to verify properties of bounded nets as in McMillan's and Probst's [10]. This could then be compared with the work of Khomenko et al. [9]. In that paper, the authors define a concise representation of a weakly safe branching process by "merging" elements with a common past, and they propose specific analysis algorithms, to exploit this concise structure in the case where the original net is safe.

In the course of the preparation of the final version of this paper, we discovered the recent work of Hayman and Winskel [6]. These authors consider nets where the support of the initial marking and the pre- and postsets of transitions may be infinite, and the marking function may take infinite values. Unfoldings are defined for these nets in a categorical setting, using classical occurrence nets and a notion of symmetries. A thorough comparison between this work and our results remains to be done.

\section{Acknowledgements}

The authors gratefully acknowledge the crucial contribution of the referees who helped eliminate technical mistakes and make the purpose of the paper clearer. 


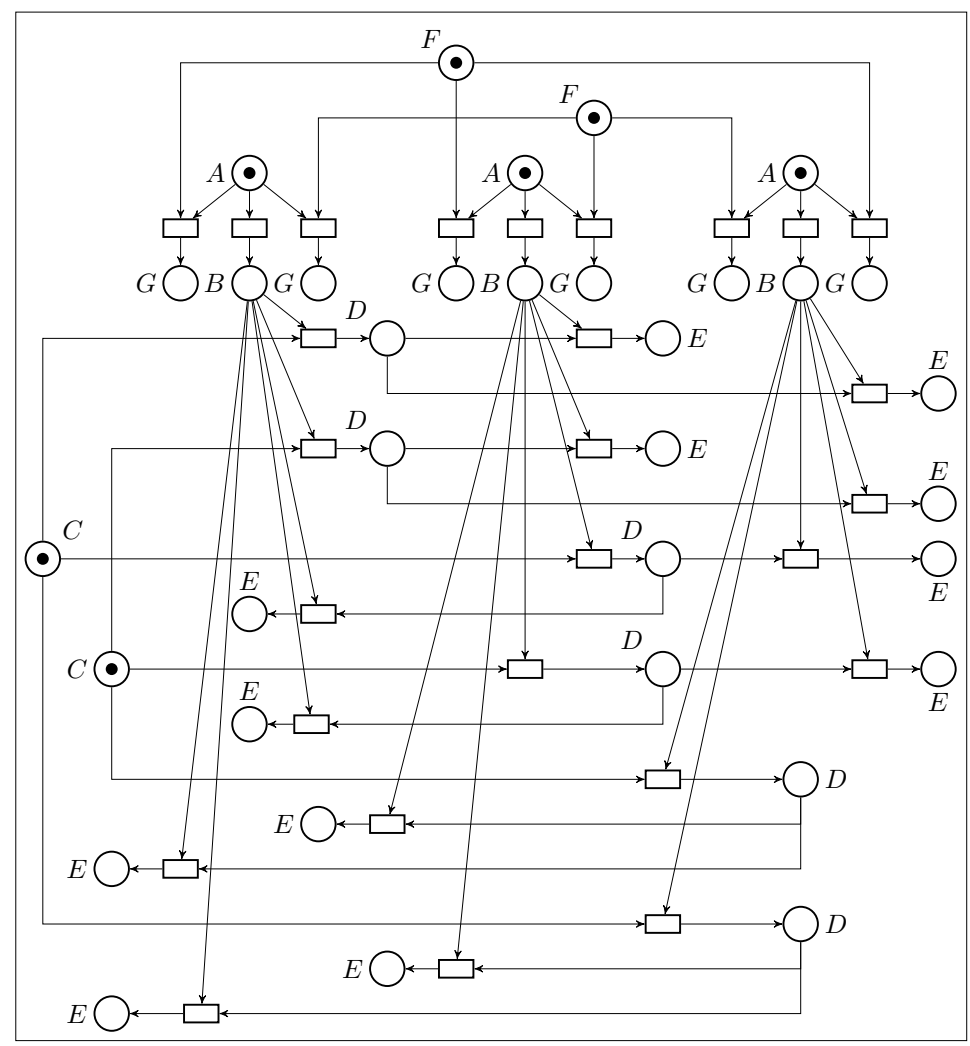

Figure 11. The weakly safe unfolding of the net $N$ in Section 6.2

\section{References}

[1] Best, E., Devillers, R.: Sequential and concurrent behaviour in Petri net theory, Theoretical Computer Science, 55, 1987, 87-136.

[2] Couvreur, J.-M., Poitrenaud, D., Weil, P.: Branching Processes of General Petri Nets, Applications and Theory of Petri Nets, 6709, Springer, 2011, 129-148.

[3] Engelfriet, J.: Branching Processes of Petri Nets, Acta Informatica, 28, 1991, 575-591.

[4] van Glabbeek, R., Plotkin, G.: Configuration structures, event structures and Petri nets, Theoretical Computer Science, 410(41), September 2009, 4111-4159.

[5] Haar, S.: Branching Processes of General S/T-Systems and their Properties, Electronic Notes in Theoretical Computer Science, 18, 1998.

[6] Hayman, J., Winskel, G.: The unfolding of general Petri nets, IARCS Annual Conference on Foundations of Software Technology and Theoretical Computer Science (FSTTCS 2008) (R. Hariharan, M. Mukund, V. Vinay, Eds.), 2, Schloss Dagstuhl-Leibniz-Zentrum fuer Informatik, Dagstuhl, Germany, 2008, $223-234$.

[7] Hoogers, P., Kleijn, H., Thiagarajan, P.: An Event Structure Semantics for General Petri Nets, Theoretical Computer Science, 153, 1996, 129-170.

[8] Keller, W.: Clustering for Petri Nets, Theoretical Computer Science, 308, 2003, 145-197. 
[9] Khomenko, V., Kondratyev, A., Koutny, M., Vogler, W.: Merged processes: a new condensed representation of Petri net behaviour, Acta Informatica, 43(5), 2006, 307-330.

[10] McMillan, K. L., Probst, D. K.: A technique of state space search based on unfolding, Formal Methods in System Design, 6, 1995, 45-65.

[11] Meseguer, J., Montanari, U., Sassone, V.: On the Model of Computation of Place/Transition Petri Nets, Proc. of the 15th Int. Conference on Application and Theory of Petri Nets, 815, Springer Verlag, 1994, 16-38.

[12] Murata, T.: Petri Nets: Properties, Analysis, and Applications, Proc. of the IEEE, 77(4), 1989, 541-580.

[13] Nielsen, M., Plotkin, G., Winskel, G.: Petri Nets, Events Structures and Domains, Part I, Theoretical Computer Science, 13(1), 1981, 85-108.

[14] Vogler, W.: Executions: A New Partial-order Semantics of Petri Nets., Theoretical Computer Science, 91, 1991, 205-238. 